\title{
The Toxicology Investigators Consortium Case Registry—the 2014 Experience
}

\author{
Sean H. Rhyee ${ }^{1}$ - Lynn Farrugia ${ }^{2}$ - Sharan L. Campleman ${ }^{3}$ - Paul M. Wax ${ }^{3,4}$. \\ Jeffrey Brent ${ }^{5}$ - On behalf of the Toxicology Investigators Consortium
}

Published online: 29 September 2015

(C) American College of Medical Toxicology 2015

\begin{abstract}
The Toxicology Investigators Consortium (ToxIC) Case Registry was established in 2010 by the American College of Medical Toxicology. The Registry includes all medical toxicology consultations performed at participating sites. The Registry was queried for all cases entered between January 1 and December 31, 2014. Specific data reviewed for analysis included demographics (age, gender, ethnicity), source of consultation, reasons for consultation, agents involved in toxicological exposures, signs, symptoms, clinical findings, fatalities, and treatment. In 2014, 9172 cases were entered in the Registry across 47 active member sites. Females accounted for $51.1 \%$ of cases. The majority $(65.1 \%)$ of cases were adults between the ages of 19 and 65 . Caucasians made up the largest identified ethnic group (48.9\%). Most Registry cases originated from the inpatient setting $(93.5 \%)$, with a large majority of these consultations coming from the
\end{abstract}

Data contained in this manuscript has not been previously presented in any form.

Electronic supplementary material The online version of this article (doi:10.1007/s13181-015-0507-7) contains supplementary material, which is available to authorized users.

Sean H. Rhyee

sean.rhyee@umassmemorial.org

1 University of Massachusetts Medical School, 55 Lake Avenue North, LA-202, Worcester, MA 01655, USA

2 Hartford Hospital, 80 Seymour Street, Hartford, CT 06102, USA

3 American College of Medical Toxicology, 10645 N. Tatum Blvd., Suite 200-111, Phoenix, AZ 85028, USA

4 UT Southwestern Medical School, 5323 Harry Hines Boulevard, Dallas, TX 75390, USA

5 Toxicology Associates, 2555 South Downing Street, Denver, CO 80210 , USA emergency department or inpatient admission services. Intentional and unintentional pharmaceutical exposures continued to be the most frequent reasons for consultation, accounting for $61.7 \%$ of cases. Among cases of intentional pharmaceutical exposure, $62.4 \%$ were associated with a self-harm attempt. Non-pharmaceutical exposures accounted for $14.1 \%$ of Registry cases. Similar to the past years, non-opioid analgesics, sedative-hypnotics, and opioids were the most commonly encountered agents. Clinical signs or symptoms were noted in $81.9 \%$ of cases. There were 89 recorded fatalities $(0.97 \%)$. Medical treatment (e.g., antidotes, antivenom, chelators, supportive care) was rendered in $62.3 \%$ of cases. Patient demographics and exposure characteristics in 2014 Registry cases remain similar to prior years. The majority of consultations arose in the acute care setting (emergency department or inpatient) and involved exposures to pharmaceutical products. Among exposures, non-opioid analgesics, sedative/hypnotics, and opioids were the most frequently encountered. A majority of cases required some form of treatment, but fatalities were rare.

Keywords Poisonings · Overdose · Surveillance · Epidemiology

\section{Introduction}

The American College of Medical Toxicology (ACMT) created the Toxicology Investigators Consortium (ToxIC) in 2010 as a means to provide a tool for clinical toxicology research and toxico-surveillance [1]. Unlike other poisoning databases, ToxIC cases are prospective and based on patients seen in clinical consultation by medical toxicologists in both inpatient and ambulatory settings. Beginning with four sites in 2010, the ToxIC registry has since expanded, including eight 
sites added in 2014. Investigators from 47 active sites, involving 77 separate facilities, submitted cases in 2014. Currently, $80.7 \%$ of the active accredited medical toxicology fellowship programs in the USA participate in the ACMT ToxIC Registry. The objective of this report is to summarize the Registry's 2014 data. Cases entered from January 1, 2014 through December 31, 2014 are described in this fifth annual report for the Registry [2-5].

Since its inception, several supplemental or subregistries have been created within ToxIC. In 2014, subregistries focusing on novel drugs of abuse and metal-on-metal hip implants were added. These, in addition to existing supplemental registries studying caustic ingestion, lipid resuscitation therapy, prescription drug misuse, snake bites, and a clinical poisoning severity score, bring the total number of current subregistries active in 2014 to seven. Additional changes to the Registry data include more detailed patient demographic information and further specification of reasons for exposures to medications or other chemical substances. In 2014, 18 abstracts based on Registry data were presented at three national meetings and three manuscripts utilizing Registry data were published [5-7].

In addition to the support from ACMT, extramural funding for the Registry came from both governmental and industry sources in 2014. Government funding was provided via three National Institute of Health (NIH) grant subawards, while industry funding was in the form of an unrestricted grant from BTG International Inc. (North America) utilized for the support of the North American Snakebite Registry.

\section{Methods}

Participating investigators agree to enter data on all medical toxicology consultations into the Registry. Cases are entered on a password-protected, online data collection form. The site is maintained by ACMT with oversight by the ToxIC Registry Steering Committee. The Registry is compliant with the Health Insurance Portability and Accountability Act and does not collect any protected health information or otherwise identifying patient data fields. Registry participation is compliant with local Institutional Review Board policies and procedures, as well as the Western Institutional Review Board (WIRB). WIRB has determined that the collection protocol based on submission of de-identified data from a clinical visit under the maintenance and control of the medical toxicologist does not meet the threshold of human subjects research under federal regulation 45 CFR 46 and associated guidance.

Collected data include presenting signs, symptoms, clinical course, treatments, limited patient demographics, outcome, and the type of and reason for toxicological exposure. The term "consultation" is used in this report to describe any encounter with a medical toxicologist. Such encounters may include admission to a toxicology inpatient service or evaluation by a medical toxicologist in the emergency department, inpatient unit, or outpatient clinic. The online collection form is formatted to ensure data remains organized and easily searchable. Free-text entry fields allow caregivers to provide further detail or supplementary information. As part of the Registry's mission of providing a real-time toxico-surveillance tool, a component of the standard data form is a sentinel detection field that signals novel or unusual cases.

For this report, a search of the database was performed to identify cases recorded from January 1, 2014 through December 31, 2014. Additional data from the subregistries will be published separately.

This descriptive report summarizes case demographics, source and location of consultation, and reason for encounter and provides proportion of cases by individual agent, agent class, and treatment provided. Summary statistics for cases involving fatalities and adverse drug reactions are also described. In the following tables describing individual agent or agent classes, unless otherwise indicated, values with fewer than five occurrences were not listed as separate items, but are further grouped in "Miscellaneous." Percentages noted in tables for individual agents represent their relative proportion within their respective agent class. For clinical signs or symptoms, the tables provide the percentage of any individual signs or symptom relative to the total number of registry cases. In the detailed treatment tables, percentages for each treatment modality represent the relative frequency among the subset of cases receiving at least one type of treatment. In instances of limited data for an entire class or clinical effects (e.g., such as ten or fewer cases overall or one agent contributing the majority ( $>80 \%)$ of a class), no detailed table is presented, but information may be described in the text section or available in the Supplementary Material.

\section{Results}

Tables 1 and 2, respectively, show the state and city (country and city for non-US sites) listings of the individual institutions participating in the ACMT ToxIC Registry. Institutions varied substantially in the number of cases entered in 2014, ranging from 1 to 833 cases submitted for this reporting year. The growth in annual case counts continued in 2014 (Fig. 1). The 47 ToxIC member sites active in 2014 entered a total of 9172 cases across 77 individual clinical facilities, representing a $6.7 \%$ increase over 2013 . 
Table 1 Participating institutions providing cases in 2014-USA

\begin{tabular}{|c|c|c|}
\hline Arizona & Massachusetts & Oregon \\
\hline Phoenix & Worcester & Portland \\
\hline Banner Good Samaritan & UMass Memorial Medical Center & Doernbecher Children's Hospital \\
\hline Phoenix Children's Hospital & Michigan & Oregon Health and Science University Hospital \\
\hline California & Grand Rapids & Oregon Occupational Toxicology \\
\hline Fresno & Spectrum Health Hospitals & Pennsylvania \\
\hline UCSF Fresno Medical Center & Minnesota & Harrisburg \\
\hline Loma Linda & St. Paul & Harrisburg Hospital \\
\hline Children's Hospital Boston & Regions Hospital & JC Blair Memorial Hospital \\
\hline Los Angeles & Missouri & Philadelphia \\
\hline $\begin{array}{l}\text { University of Southern California } \\
\text { Verdugo Hills }\end{array}$ & Kansas City & Einstein Medical Center \\
\hline San Diego & Children's Mercy Hospitals \& Clinics & Hahnemann University Hospital \\
\hline Kaiser San Diego & St. Louis & Mercy Fitzgerald Hospital \\
\hline San Francisco & Washington University School of Medicine & Mercy Hospital of Philadelphia \\
\hline San Francisco General Hospital & Nebraska & St. Christopher's Hospital for Children \\
\hline Colorado & Omaha & Pittsburgh \\
\hline Denver & University of Nebraska Medical Center & UPMC Children's Hospital of Pittsburgh \\
\hline Children's Hospital Colorado & New Jersey & UPMC Magee Women’s Hospital \\
\hline Denver Health Medical Center & Morristown & UPMC Presbyterian/Shadyside \\
\hline Porter and Littleton Adventist Hospital & Morristown Medical Center & Texas \\
\hline Swedish Medical Center & New Brunswick & Dallas \\
\hline University of Colorado Medical Center & Robert Wood Johnson University Hospital & Children's Medical Center Dallas \\
\hline Connecticut & Newark & Parkland Memorial Hospital \\
\hline Hartford & New Jersey Medical School (Rutgers) & St Paul University Hospital (UT) \\
\hline Connecticut Children's Medical Center & New Mexico & University of Texas (UT) Southwestern Medical \\
\hline Hartford Hospital & Albuquerque & Houston \\
\hline John Dempsey Hospital & University of New Mexico Hospital & Ben Taub General Hospital \\
\hline Georgia & New York & Texas Children's Hospital \\
\hline Atlanta & Manhasset & San Antonio \\
\hline Grady Memorial Hospital & Long Island Jewish Medical Center & San Antonio Military Medical Center \\
\hline Illinois & North Shore University Hospital & Utah \\
\hline Chicago & Staten Island University Hospital & Salt Lake City \\
\hline UIC Medical Center & New York & Primary Children's Hospital \\
\hline Evanston & Bellevue Medical Center & University of Utah Hospital \\
\hline Evanston North Shore University Health System & Mount Sinai Hospital & Virginia \\
\hline Indiana & NYU Langone Medical Center & Charlottesville \\
\hline Indianapolis & Rochester & University of Virginia Health Systems \\
\hline IU-Indiana University Hospital & Highland Hospital & Richmond \\
\hline IU-Methodist Hospital-Indianapolis & Huther-Doyle & $\begin{array}{l}\text { Virginia Commonwealth University (VCU) } \\
\text { Medical }\end{array}$ \\
\hline IU-Riley Hospital for Children & Strong Memorial Hospital & Wisconsin \\
\hline IU-Wishard Memorial Hospital & Syracuse & Milwaukee \\
\hline Massachusetts & SUNY Upstate Medical University & Children's Hospital of Wisconsin \\
\hline Boston & North Carolina & Froedtert Memorial Lutheran Hospital \\
\hline Beth Israel Boston & $\begin{array}{l}\text { Charlotte } \\
\text { Carolinas Medical Center }\end{array}$ & \\
\hline
\end{tabular}

\section{Demographics}

Tables 3 and 4 summarize case demographic data for gender, age, race, and Hispanic ethnicity. In 2014, females comprised a slight majority of the Registry cases: 4691
$(51.1 \%)$ to 4481 (48.9\%), females to males, respectively. Sixty female cases were identified as being pregnant $(1.3 \%)$, accounting for $0.7 \%$ of all cases. Adults between the ages of 19 and 65 comprised the majority $(65.1 \%)$ of reported cases. Adolescents (13 to 18 years) were the next 
Table 2 Participating institutions providing cases - international

Australia
Melbourne
Austin Hospital
Sydney
Sydney-Blacktown-Mt. Druitt Health
Canada
Toronto
Hospital for Sick Children
Israel
Haifa
Rambam Health Care Campus
Saudi Arabia
Riyadh
King Abdulaziz Medical City

ToxIC maintains a related Registry of other international sites not reflected in this report

most frequent age category at $17.1 \%$ of the cases. Data fields to establish race and Hispanic ethnicity information were newly introduced to the Registry in August 2014. Table 3 summarizes the available race/ethnicity data for this subset of cases $(N=4759$ field eligible). Caucasians made up the largest identified race group at $48.9 \%$.

\section{Source of Referral and Primary Reason for Encounter}

Hospital emergency departments were the most frequent source of referral, accounting for 5607 (61.1\%) of all Registry cases combined. The vast majority of all ToxIC cases (93.5\%) were seen in the emergency department (ED) or as an inpatient (IP) $(N=8570)$, with an order of magnitude fewer seen as outpatients $(N=602$ or $6.6 \%)$. As shown on Table 5, ED/IP consultation referrals came via the ED ( $N=5590$ or $65.2 \%$ of $\mathrm{ED} / \mathrm{IP}$ cases), admitting services ( $N=2036$ or $23.8 \%$ ), with a limited number from outside hospital transfer or other hospital non-ED service request ( $N=901$ or $10.5 \%$ ). In comparison, outpatient (OP) referrals were primarily via patient selfreferral $(N=264$ or $43.9 \%$ of OP cases) or primary care/

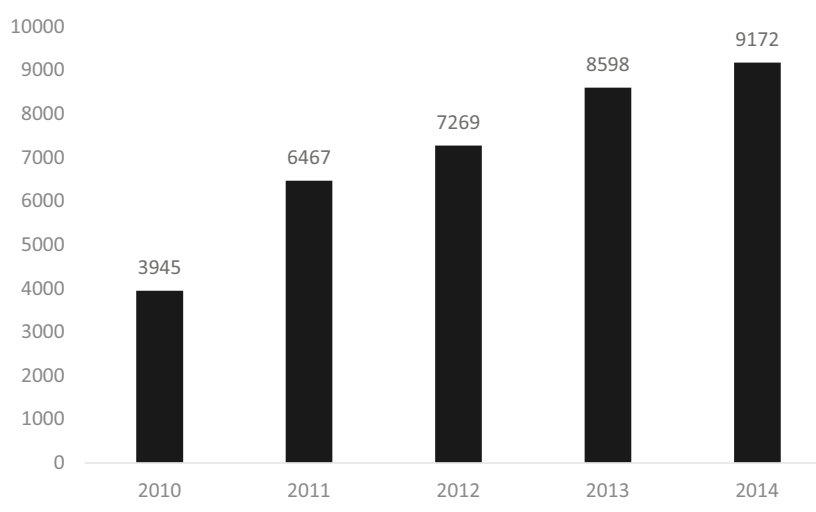

Fig. 1 ACMT ToxIC Registry total case count by year, 2010-2014
Table 3 ToxIC case demographics - age and gender

\begin{tabular}{ll}
\hline & $N(\%)$ \\
\hline Gender & \\
Male & \\
Female & $4481(48.9)$ \\
Pregnant & $4691(51.1)$ \\
Age (years) & $60(0.7)$ \\
$2-6$ & \\
$7-12$ & $442(4.8)$ \\
$13-18$ & $243(2.6)$ \\
$19-65$ & $1567(17.1)$ \\
$66-89$ & $5968(65.1)$ \\
$>89$ & $548(6.0)$ \\
Unknown & $29(0.3)$ \\
Total & $29(0.3)$ \\
\hline
\end{tabular}

other provider referrals $(N=221$ or $36.7 \%)$. Poison center referrals accounted for $4.8 \%$ of the OP and $0.1 \%$ of the $\mathrm{ED} / \mathrm{IP}$ referrals in 2014.

Exposure to pharmaceutical products, both intentional and unintentional, was the most common reason for consultation, accounting for $61.7 \%$ of all consultations (Table 6 ). By comparison, exposure to non-pharmaceuticals accounted for $14.1 \%$. All types of withdrawal combined were reported as the primary reason for encounter in $6.3 \%$ or 575 cases, while all types of envenomation resulted $3.3 \%$ or 304 cases. In 2014, additional data fields were added to the Registry for cases of intentional pharmaceutical exposure in order to further specify the presence of self-harm or suicidal intent. Within this subset of 4802 cases, self-harm attempt was reported in

Table 4 ToxIC case demographics - race and Hispanic ethnicity

\begin{tabular}{ll}
\hline & $N(\%)$ \\
\hline Race & \\
Caucasian & $2369(48.9)$ \\
Unknown/uncertain & $1563(32.8)$ \\
Black/African & $449(9.4)$ \\
Other & $230(4.8)$ \\
Asian & $86(1.8)$ \\
Multiple & $51(1.1)$ \\
American Indian/Alaska Native & $48(1.0)$ \\
Australian Aboriginal & $<5(<0.1)$ \\
Native Hawaiian or Pacific Islander & $<5(<0.1)$ \\
Hispanic ethnicity & \\
Hispanic & $450(9.5)$ \\
Non-Hispanic & $2654(55.8)$ \\
Unknown & $1402(29.5)$ \\
Total & $9172(100)$ \\
\hline
\end{tabular}

Race/ethnicity counts and frequency derived from 4759 cases with available data after July 2014 (51.9 \% of the total number of cases in 2014)

${ }^{\text {a }}$ Hispanic ethnicity as indicated exclusive of race 
Table 5 ToxIC registry case referral sources by inpatient/outpatient status

\begin{tabular}{ll}
\hline & $N(\%)$ \\
\hline Emergency department (ED) or inpatient (IP) ${ }^{\mathrm{a}}$ & \\
ED & $5590(65.2)$ \\
Admitting service & $2036(23.8)$ \\
Outside hospital transfer & $632(7.4)$ \\
Request from another hospital service (not ED) & $269(3.1)$ \\
Primary care provider/other OP treating physician & $26(0.3)$ \\
Poison center & $12(0.1)$ \\
Employer/independent med evaluation/workman's comp & $<5(<0.1)$ \\
Self-referral & $<5(<0.1)$ \\
ED/IP total & $8570(100)$ \\
Outpatient (OP)/clinic/office consultation ${ }^{\mathrm{b}}$ & \\
Self-referral & $264(43.9)$ \\
Primary care provider or other OP treating physician & $221(36.7)$ \\
Employer/independent med eval/workman's comp & $65(10.8)$ \\
Poison center & $29(4.8)$ \\
ED & $17(2.8)$ \\
Request from another hospital service (not ED) & $<5(<0.9)$ \\
Admitting service & $<5(<0.9)$ \\
OP total & $602(100)$ \\
\hline
\end{tabular}

${ }^{\text {a }}$ Percentage based on the total number of cases $(N=8570)$ seen by a medical toxicologist as consulting (ED or IP) or as attending (IP)

${ }^{\mathrm{b}}$ Percentage based on the total number of cases $(N=602)$ seen by a medical toxicologist as outpatient, clinic visit, or office consultation

2996 (62.4\%), with suicidal intent reported in 2327 (48.4 \%) (Table 7).

\section{Agent Classes}

A total of 12,496 individual agents were listed among the 8040 Registry cases reporting a toxicological exposure. Reporting medical toxicologists indicated no suspected toxicological exposure in the remainder of cases $(12.4 \%)$. The distribution of these agents among the Registry's 40 predefined substance classes is shown in Table 8. Exposure to more than one agent was reported in 2755 (30.0\%) of cases. Similar to 2013 Registry data, non-opioid analgesics, sedative/ hypnotic agents, opioids, and antidepressants constituted the most commonly encountered substance classes, collectively accounting for nearly one half (46.1\%) of all agents reported in 2014. Eight agent classes contributed to $37.7 \%$ of the total cases: ethanol (6.8\%), anticholinergic/antihistamine $(6.1 \%)$, cardiovascular $(5.7 \%)$, antipsychotic (5.5\%), sympathomimetic (5.5\%), anticonvulsant (3.4\%), psychoactive (2.5\%), and envenomation (2.3\%). Table 8 provides comparative data from prior years of the Registry (2010-2013), again as the case number and relative frequency by agent class. Several classes have been added since 2010 as noted by "NR" in earlier years, including both pharmaceuticals (e.g., cough and cold,
Table 6 Reasons for medical toxicology encounter/consultation

\begin{tabular}{|c|c|}
\hline & $N(\%)$ \\
\hline Intentional exposure - pharmaceutical & $4803(52.4)$ \\
\hline Intentional exposure-non-pharmaceutical & $913(10.0)$ \\
\hline Unintentional exposure - pharmaceutical & $853(9.3)$ \\
\hline Unintentional exposure - non-pharmaceutical & $379(4.1)$ \\
\hline Organ system dysfunction & $347(3.8)$ \\
\hline Not documented & $297(3.2)$ \\
\hline Withdrawal—opioids & $270(2.9)$ \\
\hline Envenomation-snake & $234(2.6)$ \\
\hline Withdrawal—ethanol & $227(2.5)$ \\
\hline Ethanol abuse & $194(2.1)$ \\
\hline Interpretation of toxicology data & $180(2.0)$ \\
\hline Environmental evaluation & $162(1.8)$ \\
\hline Occupational evaluation & $130(1.4)$ \\
\hline Withdrawal—sedative/hypnotic & $51(0.6)$ \\
\hline Envenomation—spider & $46(0.5)$ \\
\hline Malicious/criminal & $27(0.3)$ \\
\hline Withdrawal—other & $19(0.2)$ \\
\hline Envenomation-scorpion & $15(0.2)$ \\
\hline Envenomation-other & $9(0.1)$ \\
\hline Withdrawal—cocaine/amphetamine & $8(0.1)$ \\
\hline Marine & $7(0.1)$ \\
\hline Adverse drug reaction & $<5(<0.01)$ \\
\hline Total & $9172(100)$ \\
\hline
\end{tabular}

anticoagulant, other pharmaceutical, Parkinson's medication) and non-pharmaceuticals (e.g., household products, rodenticides).

Table 7 Detailed reasons for encounter-intentional pharmaceutical exposure

$$
N(\%)
$$

Reason for intentional pharmaceutical exposure subgroup ${ }^{\mathrm{a}}$ Attempt at self-harm

$2996(62.4)$

Abuse/misuse

$796(16.6)$

Therapeutic use

$465(9.7)$

Unknown

$384(8.0)$

None listed

Attempt at self-harm—suicidal intent subclassification ${ }^{\mathrm{b}}$

Suicidal intent

$2327(77.7)$

Suicidal intent unknown

$277(9.2)$

No data entered for suicidal intent 259 (8.6)

No data entered for suicidal intent 133 (4.4)

$2996(100)$

\footnotetext{
${ }^{\text {a }}$ Percentage of total number of cases $(N=4802)$ indicating primary reason for encounter due to intentional pharmaceutical exposure

${ }^{\mathrm{b}}$ Percentage of number of cases indicating attempt at self-harm $(N=2996)$
} 
Table 8 Agent classes involved in medical toxicology consultation

\begin{tabular}{|c|c|c|c|c|c|}
\hline & $\begin{array}{l}2014 \\
N(\%)\end{array}$ & $\begin{array}{l}2013 \\
N(\%)\end{array}$ & $\begin{array}{l}2012 \\
N(\%)\end{array}$ & $\begin{array}{l}2011 \\
N(\%)\end{array}$ & $\begin{array}{l}2010 \\
N(\%)\end{array}$ \\
\hline Analgesic (nonopioid) & $1599(12.8)$ & $1490(13.2)$ & $1295(12.3)$ & $1368(12.3)$ & $854(14.8)$ \\
\hline Sedative-hypnotic/muscle relaxant & $1546(12.4)$ & $1383(12.3)$ & $1422(13.5)$ & $1492(13.4)$ & $783(13.6)$ \\
\hline Opioid & $1311(10.5)$ & $1250(11.1)$ & $1086(10.3)$ & $1100(9.9)$ & $619(10.7)$ \\
\hline Antidepressant & $1301(10.4)$ & $1056(9.4)$ & $1039(9.8)$ & $1029(9.3)$ & $659(11.4)$ \\
\hline Ethanol & $849(6.8)$ & $737(6.5)$ & $850(8.1)$ & $580(5.2)$ & $371(6.4)$ \\
\hline Anticholinergic/antihistamine & $761(6.1)$ & $617(5.5)$ & $457(4.3)$ & $549(4.9)$ & $378(6.5)$ \\
\hline Cardiovascular & $713(5.7)$ & $687(6.1)$ & $616(5.8)$ & $631(5.7)$ & $334(5.8)$ \\
\hline Antipsychotic & $689(5.5)$ & $626(5.6)$ & $551(5.2)$ & $587(5.3)$ & $366(6.3)$ \\
\hline Sympathomimetic & $684(5.5)$ & $702(6.2)$ & $692(6.6)$ & $774(7.0)$ & $247(4.3)$ \\
\hline Anticonvulsant & $421(3.4)$ & $408(3.6)$ & $339(3.2)$ & $451(4.1)$ & $218(3.8)$ \\
\hline Psychoactive & $312(2.5)$ & $302(2.7)$ & $460(4.4)$ & $360(3.2)$ & $135(2.3)$ \\
\hline Envenomation & $282(2.3)$ & $188(1.7)$ & $196(1.9)$ & $183(1.6)$ & $105(1.8)$ \\
\hline Diabetic medications & $210(1.7)$ & $181(1.6)$ & $138(1.3)$ & $113(1.0)$ & $65(1.1)$ \\
\hline Lithium & $179(1.4)$ & $166(1.5)$ & $133(1.3)$ & $100(0.9)$ & $78(1.4)$ \\
\hline Cough and cold products & $161(1.3)$ & $134(1.2)$ & NR & NR & NR \\
\hline Herbal products/dietary supplements & $159(1.3)$ & $119(1.1)$ & $50(0.5)$ & $76(0.7)$ & $48(0.8)$ \\
\hline Metals & $145(1.2)$ & $154(1.4)$ & $227(2.2)$ & $322(2.9)$ & $154(2.7)$ \\
\hline Gases/irritants/vapors/dusts & $138(1.1)$ & $126(1.1)$ & $129(1.2)$ & $169(1.5)$ & $63(1.1)$ \\
\hline Household product & $125(1.0)$ & $113(1.0)$ & NR & NR & NR \\
\hline Unknown agent & $109(0.9)$ & $88(0.8)$ & NR & NR & NR \\
\hline Antimicrobial & $104(0.8)$ & $113(1.0)$ & $62(0.6)$ & $107(1.0)$ & $38(0.7)$ \\
\hline Toxic alcohol & $104(0.8)$ & $95(0.8)$ & $121(1.1)$ & $145(1.3)$ & $93(1.6)$ \\
\hline Hydrocarbon & $84(0.7)$ & $84(0.8)$ & $45(0.4)$ & $67(0.6)$ & $50(0.9)$ \\
\hline Caustic & $80(0.6)$ & $88(0.8)$ & $47(0.4)$ & $93(0.8)$ & $45(0.8)$ \\
\hline Plants and fungi & $75(0.6)$ & $71(0.6)$ & $52(0.5)$ & $78(0.7)$ & $18(0.3)$ \\
\hline Anticoagulant & $64(0.5)$ & $58(0.5)$ & $\mathrm{NR}$ & NR & NR \\
\hline Endocrine & $43(0.3)$ & $34(0.3)$ & $49(0.5)$ & $37(0.3)$ & $9(0.2)$ \\
\hline Other non-pharmaceutical product & $39(0.3)$ & $14(0.1)$ & NR & NR & NR \\
\hline Chemotherapeutic/immunological & $37(0.3)$ & $23(0.2)$ & $12(0.1)$ & $20(0.2)$ & $5(0.1)$ \\
\hline Rodenticide & $35(0.3)$ & $15(0.1)$ & $\mathrm{NR}$ & NR & NR \\
\hline Gastrointestinal agents & $33(0.3)$ & $34(0.3)$ & $30(0.3)$ & $50(0.4)$ & $14(0.2)$ \\
\hline Insecticide & $30(0.2)$ & $27(0.2)$ & NR & NR & NR \\
\hline Other pharmaceutical product & $25(0.2)$ & $30(0.3)$ & NR & NR & NR \\
\hline Anesthetic & $19(0.2)$ & $11(0.1)$ & $30(0.3)$ & $21(0.2)$ & $16(0.3)$ \\
\hline Anti-parkinsonism drugs & $9(0.1)$ & $19(0.2)$ & NR & NR & NR \\
\hline Pulmonary & $9(0.1)$ & $7(0.1)$ & $16(0.2)$ & $17(1.5)$ & $9(0.2)$ \\
\hline Ingested foreign object & $6(0.05)$ & $<5(<0.03)$ & NR & NR & NR \\
\hline Herbicide & $5(0.04)$ & $11(0.1)$ & NR & NR & NR \\
\hline Fungicide & $<5(<0.03)$ & $<5(<0.03)$ & $\mathrm{NR}$ & NR & NR \\
\hline WMD/riot agent/radiological & $<5(<0.03)$ & $<5(<0.03)$ & $<5(<0.03)$ & $7(0.1)$ & $0(0.0)$ \\
\hline Total annual agent entries & 12,496 & 11,279 & 10,553 & 11,119 & 5774 \\
\hline
\end{tabular}

Percentages are out of the total number of reported agent entries per year; $30 \%$ of 2014 Registry cases reported exposure to multiple agents $N R$ no cases reported, class category not available

\section{Individual Agents by Class}

Tables 9, 10, 11, 12, 13, 14, 15, 16, 17, 18, 19, 20, 21, 22, 23, 24 , and 25 summarize specific pharmaceuticals and other substances reported as a toxic exposure by class, in order, based on relative contribution to the total number of agents reported to the Registry, with two exceptions. Single agent classes for ethanol and lithium are instead presented and discussed with toxic alcohols and anticonvulsants, respectively. Additional detailed information for agent classes with relatively smaller 
Table 9 Analgesics

\begin{tabular}{ll}
\hline & $N(\%)$ \\
\hline Acetaminophen & $1051(64.6)$ \\
Aspirin & $270(16.9)$ \\
Ibuprofen & $194(12.1)$ \\
Naproxen & $52(3.3)$ \\
Salicylamide & $8(0.5)$ \\
Meloxicam & $7(0.4)$ \\
Methylsalicylate & $5(0.3)$ \\
Miscellaneous $^{\mathrm{a}}$ & $12(0.8)$ \\
Class total & $1599(100)$ \\
\hline
\end{tabular}

${ }^{a}$ Includes ketorolac, NSAID unspecified, phenazopyridine, analgesic unspecified, diclofenac, indomethacin, metamizole, piroxicam, and salsalate

case numbers and/or individual agents may be found in the online Supplementary Material.

Non-opioid analgesic entries for 2014 are shown in Table 9. Acetaminophen exposures accounted for $11.5 \%$ of all Registry cases in 2014 and were the most common analgesic exposure (64.6\% within class frequency). Non-salicylate NSAIDS made up $24.6 \%$ of this category, with ibuprofen the most common (12.1\%). Salicylates made up $27.0 \%$ of the cases, primarily involving aspirin (16.9\%).

Sedative-hypnotic agents and muscle relaxants accounted for $12.4 \%$ of all the agents reported (Table 8 ). As summarized in Table 10, benzodiazepines at $54.5 \%$ accounted for the majority of the class as a whole, followed by muscle relaxants (20.7\%), other sedatives (17.4\%), nonbenzodiazepine agonists $(8.9 \%)$, and barbiturates (3.4\%). The two most common benzodiazepines, clonazepam (20.7\%) and alprazolam $(15.1 \%)$, accounted for over one third of the class. At least one benzodiazepine was reported in $9.1 \%$ of all Registry cases. Zolpidem was the most common nonbenzodiazepine agent reported in this overall class (8.2\%), followed closely by the muscle relaxant cyclobenzaprine $(7.5 \%)$ and sedative gabapentin $(7.4 \%)$.

Table 11 summarizes the class of opioids, a category including natural opiates, semisynthetic and synthetic opioid agents. In 2014, 1311 individual opioid agents were reported, just over $10 \%$ of all agents (Table 8 ). As in previous years, semisynthetic agents (heroin, oxycodone, hydrocodone, buprenorphine, hydromorphine, and oxymorphone) were the most common class subset at $63.3 \%$ of all opioid entries. Heroin was the most common semisynthetic agent, at $26.7 \%$. Oxycodone was also relatively common, at $18.6 \%$ of the class. The synthetic opioids (methadone, tramadol, fentanyl, naltrexone, loperamide, and naloxone) accounted for $25.7 \%$ of the overall class, primarily due to methadone, tramadol, and fentanyl. The most common synthetic was methadone $(12.1 \%)$. The opiates morphine and codeine accounted for $5.7 \%$ of the class.
Table 10 Sedative-hypnotics/muscle relaxants by subtype

\begin{tabular}{|c|c|}
\hline & $N(\%)$ \\
\hline Benzodiazepines & $843(54.5)$ \\
\hline Clonazepam & $320(20.7)$ \\
\hline Alprazolam & $234(15.1)$ \\
\hline Lorazepam & $127(8.2)$ \\
\hline Diazepam & $80(5.2)$ \\
\hline Benzodiazepine unspecified & $36(2.3)$ \\
\hline Temazepam & $20(1.3)$ \\
\hline Chlordiazepoxide & $13(0.8)$ \\
\hline Miscellaneous $^{\mathrm{a}}$ & $13(0.8)$ \\
\hline Muscle relaxants & $320(20.7)$ \\
\hline Cyclobenzaprine & $116(7.5)$ \\
\hline Carisoprodol & $78(5.0)$ \\
\hline Baclofen & $76(4.9)$ \\
\hline Tizanidine & $24(1.6)$ \\
\hline Methocarbamol & $10(0.6)$ \\
\hline Metaxalone & $6(0.4)$ \\
\hline Miscellaneous $^{\mathrm{b}}$ & $10(0.6)$ \\
\hline Other sedatives & $193(12.5)$ \\
\hline Gabapentin & $115(7.4)$ \\
\hline Pregabalin & $35(2.3)$ \\
\hline Buspirone & $23(1.5)$ \\
\hline Sedative-hypnotic/muscle relaxant unspecified & $9(0.6)$ \\
\hline Propofol & $7(0.5)$ \\
\hline Miscellaneous ${ }^{\mathrm{c}}$ & $<5(<0.4)$ \\
\hline Non-benzodiazepine agonists (“Z” drugs) & $138(8.9)$ \\
\hline Zolpidem & $126(8.2)$ \\
\hline Eszopiclone & $7(0.5)$ \\
\hline Miscellaneous ${ }^{\mathrm{d}}$ & $5(0.3)$ \\
\hline Barbiturates & $52(3.4)$ \\
\hline Butalbital & $37(2.4)$ \\
\hline Phenobarbital & $12(0.8)$ \\
\hline Miscellaneous $^{\mathrm{e}}$ & $<5(<0.3)$ \\
\hline Class total & $1546(100)$ \\
\hline
\end{tabular}

a Includes midazolam, nitrazepam, bromazepam, oxazepam, etizolam, and flunitrazepam

${ }^{\mathrm{b}}$ Includes meprobamate, chlorzoxazone, and orphenadrine

${ }^{\mathrm{c}}$ Includes phenibut, chlorbutol, and ramelteon

${ }^{\mathrm{d}}$ Includes zopiclone and zaleplon

${ }^{\mathrm{e}}$ Includes pentobarbital and butabarbital

Antidepressants accounted for $10.4 \%$ of the total agents reported in the Registry (Table 8). As shown in Table 12, the two most common individual antidepressants reported were bupropion $(17.7 \%)$ and trazodone (13.8\% class). By comparison, the tricyclic antidepressants combined (13.5\%) appeared at a considerable lower frequency. Over one third of the antidepressants reported were selective serotonin reuptake inhibitors (SSRIs). The most common SSRI, citalopram, accounted for $10.4 \%$ of the antidepressant agents reported. 
Table 11 Opioids

\begin{tabular}{ll}
\hline & $N(\%)$ \\
\hline Heroin & $350(26.7)$ \\
Oxycodone & $244(18.6)$ \\
Methadone & $158(12.1)$ \\
Hydrocodone & $128(9.8)$ \\
Tramadol & $124(9.5)$ \\
Buprenorphine & $80(6.1)$ \\
Opioid unspecified & $54(4.1)$ \\
Morphine & $52(4.0)$ \\
Fentanyl & $38(2.9)$ \\
Codeine & $30(2.3)$ \\
Hydromorphone & $15(1.1)$ \\
Oxymorphone & $12(0.9)$ \\
Naltrexone & $7(0.5)$ \\
Loperamide & $5(0.4)$ \\
Naloxone & $5(0.4)$ \\
Miscellaneous & $9(0.7)$ \\
Class total & $1311(100)$ \\
\hline
\end{tabular}

a Includes tapentadol, diphenoxylate, desomorphine, and papaverine
Table 13 Anticholinergics and antihistamines

\begin{tabular}{ll}
\hline & $N(\%)$ \\
\hline Diphenhydramine & $406(53.4)$ \\
Hydroxyzine & $110(14.5)$ \\
Doxylamine & $45(5.9)$ \\
Chlorpheniramine & $42(5.5)$ \\
Benztropine & $37(4.9)$ \\
Promethazine & $26(3.4)$ \\
Cetirizine & $16(2.1)$ \\
Loratadine & $13(1.7)$ \\
Antihistamine unspecified & $7(0.9)$ \\
Trihexyphenidyl & $7(0.9)$ \\
Dimenhydrinate & $6(0.8)$ \\
Oxybutynin & $6(0.8)$ \\
Anticholinergic unspecified & $5(0.6)$ \\
Dicyclomine & $5(0.6)$ \\
Meclizine & $5(0.6)$ \\
Miscellaneous & a \\
Class total & $25(3.3)$ \\
\hline
\end{tabular}

${ }^{a}$ Includes fexofenadine, hyoscyamine, atropine, brompheniramine, scopolamine, belladonna, fesoterodine, pheniramine, pyrilamine, tiotropium, and tolterodine

Table 12 Antidepressants

\begin{tabular}{ll}
\hline & $N(\%)$ \\
\hline Other antidepressants & $485(37.3)$ \\
Bupropion & $230(17.7)$ \\
Trazodone & $180(13.8)$ \\
Mirtazapine & $62(4.8)$ \\
Vilazodone & $6(0.5)$ \\
Miscellaneous ${ }^{\mathrm{a}}$ & $7(0.5)$ \\
Selective serotonin reuptake inhibitors (SSRIs) & $480(36.9)$ \\
Citalopram & $135(10.4)$ \\
Sertraline & $114(8.8)$ \\
Fluoxetine & $109(8.4)$ \\
Escitalopram & $78(6.0)$ \\
Paroxetine & $44(3.4)$ \\
Tricyclic antidepressants (TCAs) & $176(13.5)$ \\
Amitriptyline & $129(9.9)$ \\
Nortriptyline & $20(1.5)$ \\
Doxepin & $18(1.4)$ \\
Imipramine & $5(0.4)$ \\
Miscellaneous ${ }^{\text {b }}$ & $<5(0.1)$ \\
Serotonin-norepinephrine reuptake inhibitors (SNRIs) & $150(11.5)$ \\
Venlafaxine & $89(6.8)$ \\
Duloxetine & $47(3.6)$ \\
Desvenlafaxine & $7(0.5)$ \\
Fluvoxamine & $7(0.5)$ \\
Class total & $1301(100)$ \\
& \\
&
\end{tabular}

${ }^{a}$ Includes antidepressant unspecified, nefazodone, tianeptine, and tranylcypromine

${ }^{\mathrm{b}}$ Includes clomipramine and desipramine

Table 8 shows that $6.1 \%$ of all agents reported in 2014 were anticholinergic/antihistamine agents. As seen in Table 13, diphenhydramine was the most common individual agent, equaling $53.4 \%$ of all reported agents in this class, followed by hydroxyzine (14.5\%), then three agents accounting for 5-6\% of the class (doxylamine, chlorpheniramine, and benzotropine). Of note, hydroxyzine was represented in $4.4 \%$ of all Registry cases.

As listed in Table 14, the most common groups of cardiovascular agents were beta blockers (27.5\%), sympatholytics (22.4\%), and calcium channel antagonists (15.0\%). Metoprolol and propranolol were the two most common beta blockers, accounting for 10.7 and $6.9 \%$, respectively. Clonidine was the most commonly reported sympatholytic, responsible for $18.2 \%$ of all cardiovascular agents, while amlodipine was the most common calcium channel antagonist (7.4\%). Clonidine was reported in $1.4 \%$ of all Registry cases. Digoxin was the predominate cardiac glycoside (69 of 70 cases). Ace inhibitors, diuretics, antidysrhythmics, and other antihypertensives and vasodilators appeared to a much lesser extent. However, lisinopril, the most common ACE inhibitor, accounted for $7.4 \%$ of the cardiovascular class.

Antipsychotics contributed $5.5 \%$ of all agent entries to the Registry (Table 8). Atypical antipsychotics represented over $83 \%$ of the class, primarily due to quetiapine $(48.0 \%)$, risperidone $(11.6 \%)$, aripiprazole $(11.0 \%)$, and olanzapine $(10.6 \%)$ (Table 15). Quetiapine exposure was represented in $6.3 \%$ of the Registry cases. Within the overall class, an array 
Table 14 Cardiovascular agents by subtype

\begin{tabular}{|c|c|}
\hline & $N(\%)$ \\
\hline Beta blockers & $196(27.5)$ \\
\hline Metoprolol & $76(10.7)$ \\
\hline Propranolol & $49(6.9)$ \\
\hline Carvedilol & $27(3.8)$ \\
\hline Atenolol & $23(3.2)$ \\
\hline Labetalol & $8(1.1)$ \\
\hline Nadolol & $6(0.8)$ \\
\hline Miscellaneous $^{\mathrm{a}}$ & $7(1.0)$ \\
\hline Sympatholytics & $160(22.4)$ \\
\hline Clonidine & $130(18.2)$ \\
\hline Guanfacine & $30(4.2)$ \\
\hline Calcium channel antagonists & $107(15.0)$ \\
\hline Amlodipine & $53(7.4)$ \\
\hline Diltiazem & $28(3.9)$ \\
\hline Verapamil & $21(2.9)$ \\
\hline Miscellaneous $^{\mathrm{b}}$ & $5(0.7)$ \\
\hline Cardiac glycosides & $70(9.8)$ \\
\hline Digoxin & $69(9.7)$ \\
\hline Digitoxin & $<5(<0.2)$ \\
\hline ACE inhibitors & $57(8.0)$ \\
\hline Lisinopril & $53(7.4)$ \\
\hline Miscellaneous $^{\mathrm{c}}$ & $5(0.6)$ \\
\hline Diuretics & $44(6.2)$ \\
\hline Hydrochlorothiazide & $21(2.9)$ \\
\hline Furosemide & $13(1.8)$ \\
\hline Miscellaneous $^{\mathrm{d}}$ & $10(1.4)$ \\
\hline Other antihypertensives and vasodilators & $28(3.9)$ \\
\hline Prazosin & $10(1.4)$ \\
\hline Miscellaneous $^{\mathrm{e}}$ & $18(2.5)$ \\
\hline Antidysrhythmics & $20(2.8)$ \\
\hline Amiodarone & $6(0.8)$ \\
\hline Flecainide & $5(0.7)$ \\
\hline Miscellaneous ${ }^{\mathrm{f}}$ & $9(1.3)$ \\
\hline Other cardiovascular agents & $17(2.4)$ \\
\hline Simvastatin & $10(1.4)$ \\
\hline Miscellaneous $^{\mathrm{g}}$ & $7(1.0)$ \\
\hline Angiotensin receptor blockers & $15(2.1)$ \\
\hline Losartan & $8(1.1)$ \\
\hline Valsartan & $5(0.7)$ \\
\hline Miscellaneous $^{\mathrm{h}}$ & $<5(<0.4)$ \\
\hline Class total & $713(100)$ \\
\hline
\end{tabular}

${ }^{a}$ Includes nebivolol and timolol

${ }^{\mathrm{b}}$ Includes nifedipine and felodipine

${ }^{\mathrm{c}}$ Includes benazepril, perindopril, and quinapril

${ }^{\mathrm{d}}$ Includes chlorthalidone, acetazolamide, bumetanide, spironolactone, and torsemide

${ }^{\mathrm{e}}$ Includes tamsulosin, isosorbide, antihypertensive unspecified, hydralazine, terazosin, alfuzosin, cilostazol, doxazosin, minoxidil, and nitroglycerin

${ }^{\mathrm{f}}$ Includes propafenone, mexiletine, dofetilide, and dronederone

${ }^{\mathrm{g}}$ Includes atorvastatin, colesevalm, fenofibrate, lovastatin, and ranolazine

${ }^{\mathrm{h}}$ Includes olmesartan and telmisartan
Table 15 Antipsychotics

\begin{tabular}{ll}
\hline & $N(\%)$ \\
\hline Quetiapine & $331(48.0)$ \\
Risperidone & $80(11.6)$ \\
Aripiprazole & $76(11.0)$ \\
Olanzapine & $73(10.6)$ \\
Haloperidol & $35(5.1)$ \\
Clozapine & $23(3.3)$ \\
Ziprasidone & $19(2.8)$ \\
Chlorpromazine & $18(2.6)$ \\
Paliperidone & $9(1.3)$ \\
Lurasidone & $8(1.2)$ \\
Miscellaneous & \\
Class total & $17(2.2)$ \\
\hline
\end{tabular}

${ }^{a}$ Includes prochlorperazine, fluphenazine, loxapine, antipsychotic unspecified, trifluoperazine, asenapine, iloperidone, and thioridazine

of "first-generation" phenothiazines resulted in $3.5 \%$ of agent entries.

Table 16 shows agents reported as sympathomimetics, including a range of pharmaceuticals, illicit drugs, and other designer stimulants associated with toxic exposures/consultations. Overall, this class contributed $5.5 \%$ of all agent entries in 2014 (Table 8). At $37.1 \%$, cocaine was the majority contributor to this class, followed by methamphetamine and amphetamine, which combined accounted for another $32.6 \%$. Pharmaceutical stimulants of interest, such as those for

Table 16 Sympathomimetics

\begin{tabular}{|c|c|}
\hline & $N(\%)$ \\
\hline Cocaine & $252(37.1)$ \\
\hline Methamphetamine & $145(21.3)$ \\
\hline Amphetamine & $77(11.3)$ \\
\hline Methylphenidate & $50(7.4)$ \\
\hline Dextroamphetamine & $33(4.9)$ \\
\hline Methylenedioxy- $N$-methamphetamine & $26(3.8)$ \\
\hline Lisdexamfetamine & $18(2.6)$ \\
\hline Phenylephrine & $15(2.2)$ \\
\hline Phentermine & $14(2.1)$ \\
\hline Sympathomimetic unspecified & $10(1.5)$ \\
\hline 25I-NBOMe & $6(0.9)$ \\
\hline Atomoxetine & $6(0.9)$ \\
\hline Cathinone & $6(0.9)$ \\
\hline Dexmethylphenidate & $6(0.9)$ \\
\hline Miscellaneous $^{\mathrm{a}}$ & $20(2.9)$ \\
\hline Class total & $684(100)$ \\
\hline
\end{tabular}

${ }^{\mathrm{a}}$ Includes clenbuterol, 2C series drugs, pseudoephedrine, epinephrine, alpha-pyrrolidinopentiophenone, ephedrine, ethylphenidate, methylenedioxypyrovalerone (MDPV), methylenedioxyethylamphetamine (MDEA), phendimetrazine, phenylethylamine designer drugs, and tetrahydrozoline 
Table 17 Anticonvulsants and mood stabilizers

\begin{tabular}{lc}
\hline & $N(\%)$ \\
\hline Lithium $^{\mathrm{a}}$ & $179(100)$ \\
Valproic acid & $116(27.6)$ \\
Lamotrigine & $94(22.3)$ \\
Phenytoin & $59(14.0)$ \\
Carbamazepine & $54(12.8)$ \\
Topiramate & $42(10.0)$ \\
Oxcarbazepine & $26(6.2)$ \\
Levetiracetam & $16(3.8)$ \\
Zonisamide & $7(1.7)$ \\
Miscellaneous & $7(1.7)$ \\
Class total & $421(100)$ \\
\hline a ${ }^{\text {bithium is considered a separate agent }}$ \\
class \\
${ }^{b}$ Includes clobazam, felbamate, and \\
lacosamide
\end{tabular}

treating attention deficit/hyperactivity disorder, contributed for $2 \%$ or more to this class. These drugs included methylphenidate $(7.4 \%)$, dextroamphetamine $(4.9 \%)$, and lisdexamfetamine $(2.6 \%)$. The most common designer amphetamine reported was methylenedioxy- $N$-methamphetamine at $3.8 \%$ of the class. A range of other designer stimulants were reported in smaller numbers, including 4-iodo-2,5dimethoxy- $N$-(2-methoxybenzyl) phenethylamine (25INBOMe), cathinone, methylenedioxypyrovalerone (MDPV), methylenedioxyethylamphetamine (MDEA), and alphapyrrolidinopentiophenone.

Anticonvulsants comprised $3.4 \%$ of the agent entries, primarily due to valproic acid (27.6\%) and lamotrigine (22.3\%) (Table 17). The mood stabilizer lithium constitutes a single agent class in the Registry. In 2014, 179 occurrences, representing $2.0 \%$ of the Registry cases, were entered.

Table 18 Psychoactives

\begin{tabular}{ll}
\hline & $N(\%)$ \\
\hline Marijuana & $101(32.4)$ \\
Cannabinoid—-synthetic & $81(26.0)$ \\
Phencyclidine & $27(8.7)$ \\
Lysergic acid diethylamide (LSD) & $25(8.0)$ \\
Nicotine & $21(6.7)$ \\
Cannabinoid-nonsynthetic & $14(4.5)$ \\
Gamma hydroxybutyrate (GHB) & $13(4.2)$ \\
Miscellaneous & $30(9.6)$ \\
Class total & $312(100)$ \\
\hline
\end{tabular}

${ }^{a}$ Includes ketamine, donepezil, ibogaine, 1,4-butanediol, dimethyltryptamine, $\gamma$-butryolactone, mephedrone, methoxetamine, psychoactive unspecified, 2,6-dimethoxy-4-methylamphetamine, Argyeria nervosa, disulfiram, hallucinogen unspecified, hallucinogenic amphetamine, methylone, tetrahydropalmatine, and vareniciline

Table19 Envenomations and marine poisonings

$N(\%)$

\begin{tabular}{ll}
\hline Crotalus spp. & $102(36.2)$ \\
Agkistrodon spp. & $80(28.4)$ \\
Snake unspecified & $30(10.6)$ \\
Loxosceles spp. & $19(6.7)$ \\
Latrodectus spp. & $18(6.4)$ \\
Centruroides spp. & $13(4.6)$ \\
Miscellaneous $^{\mathrm{a}}$ & $20(7.1)$ \\
Class total & $282(100)$
\end{tabular}

${ }^{a}$ Includes envenomation unspecified, Vipera palaesinae, scombroid poisoning, scorpion unspecified, ciguatera poisoning, hymenoptera, insect unspecified, jellyfish, Pterios spp. (lionfish), palytoxin, Scolopendra spp. (centipedes), spider unspecified, stingray, and Trimeresurus abolabris

Cases classified as involving other psychoactive drugs of abuse are shown in Table 18. Marijuana was the most frequent entry in this category in 2014, representing $32.4 \%$ of all entries in this group. Synthetic cannabinoids, such as those referred to as "spice" or "K2," were reported in $26.0 \%$ of all class entries. Other psychoactive compounds included phencyclidine, lysergic acid diethylamide (LSD), and gamma hydroxybutyrate (GHB).

Among envenomations and marine poisonings, over $70 \%$ were related to Crotalus spp. (rattlesnake), Agkistrodon spp. (water moccasin), or snake species unspecified (Table 19); $17.7 \%$ of envenomations were attributed to Loxsocoles spp. (recluse spider), Latrodectus spp. (widow spider), and Centruroides spp. (bark scorpions).

Antidiabetic medications metformin (29.5\%), insulin (24.3\%), glipizide $(18.6 \%)$, and glyburide $(15.7 \%)$ were responsible for the majority of entries among the diabetesrelated medications reported (Table 20). Dextromethorphan accounted for $87.0 \%$ of cough and cold product entries (see Supplementary Material).

Table 20 Diabetic medications

\begin{tabular}{ll}
\hline & $N(\%)$ \\
\hline Metformin & $62(29.5)$ \\
Insulin & $51(24.3)$ \\
Glipizide & $39(18.6)$ \\
Glyburide & $33(15.7)$ \\
Glimepiride & $11(5.2)$ \\
Sulfonylurea unspecified & $5(2.4)$ \\
Miscellaneous & \\
Class total & $9(4.3)$ \\
\hline
\end{tabular}

${ }^{a}$ Includes sitagliptin, pioglitazone, gliclazide, liraglutide, and repaglinide 
Table 21 Ethanol and toxic alcohols

\begin{tabular}{|c|c|}
\hline & $N(\%)$ \\
\hline Ethanol $^{\mathrm{a}}$ & $849(100.0)$ \\
\hline \multicolumn{2}{|c|}{ Nonethanol alcohols and glycols } \\
\hline Ethylene glycol & $40(38.5)$ \\
\hline Isopropanol & $29(27.9)$ \\
\hline Methanol & $14(13.5)$ \\
\hline Acetone & $9(8.7)$ \\
\hline Miscellaneous $^{\mathrm{b}}$ & $12(11.5)$ \\
\hline Class total & $104(100)$ \\
\hline \multicolumn{2}{|c|}{$\begin{array}{l}{ }^{a} \text { Ethanol is considered a separate agen } \\
\text { class }\end{array}$} \\
\hline \multicolumn{2}{|c|}{$\begin{array}{l}\text { b Includes methyl ethyl ketone, propylene } \\
\text { glycol, glycol ether unspecified, butyl eth- } \\
\text { ylene glycol, and toxic alcohol unspecified }\end{array}$} \\
\hline
\end{tabular}

Nine additional classes comprised the remaining pharmaceutical group classifications, accounting for $0.1-0.9 \%$ of all agent entries (Table 8). A large number of individual agents contributed to the 69 antibiotics, 21 antivirals, antifungals, and other types of antimicrobials (both pharmaceutical and nonpharmaceutical uses). Warfarin accounted for $71.2 \%$ of the anticoagulants, followed by rivaroxaban $(10.6 \%)$, with a variety of other agents in the class accounting for a limited number of entries (see Supplementary Material). For the remaining drug classes, the most common agents were the following: levothyroxine (endocrine agents), hydrogen peroxide and pyridostigmine (other pharmaceuticals), benzonatate (anesthetics), omeprazole (gastrointestinal), levodopa/carbodopa (anti-parkinsonism), theophylline (pulmonary), and hydroxychloroquine (chemotherapeutic and immunological).

Alcohols were classified into two categories, ethanol and other toxic alcohols (Table 21). As a single agent class, ethanol was responsible for $6.8 \%$ of all agents. Table 21

Table 22 Plants and fungi

Table 23 Metals

\begin{tabular}{lc}
\hline & $N(\%)$ \\
\hline Lead & $37(25.5)$ \\
Iron & $22(15.2)$ \\
Cobalt & $17(11.7)$ \\
Chromium & $14(9.7)$ \\
Mercury & $13(9.0)$ \\
Arsenic & $6(4.1)$ \\
Copper & $6(4.1)$ \\
Miscellaneous ${ }^{\text {a }}$ & $30(20.7)$ \\
Class total & $145(100)$ \\
\hline a Includes gadolinium, manganese, mag- \\
nesium, selenium, titanium, aluminum, \\
metal unspecified, silver, antimony, beryl- \\
lium, cadmium, cesium, thallium, urani- \\
um, and zinc sulfate
\end{tabular}

summarizes case numbers for the other, less common toxic alcohols including ethylene glycol, isopropanol, methanol, and acetone, which as a group accounted for $0.8 \%$ of the agent entries.

The agent class herbals and dietary supplements captured a broad range of products from a variety of sources (herbal, mineral, or chemical). Three single agents were responsible for $64.1 \%$ of the 159 entries: caffeine, melatonin, and multivitamins. Over 30 other agents were responsible for the remaining $35.9 \%$. Table 22 shows a similar situation for the plant and fungi class, and mold (unspecified) was the most common agent class entry ( $34.7 \%$ ), followed by mushroom unspecified (16.0 \%) and mushroom, Psilocybe spp. (8.0\%), with 22 other specific agents accounting for the remaining $41.3 \%$ (31 cases) (data in Supplementary Materials).

Eight classes of agents were most often reported in occupational and environmental exposures: metals, hydrocarbons, pesticides, gases, caustics, irritants, vapors, and dusts. In the metal class, lead, iron, and cobalt accounted for over one half

Table 24 Gases, irritants, vapors, and dusts

\begin{tabular}{ll}
\hline & $N(\%)$ \\
\hline Carbon monoxide & $81(58.7)$ \\
Cyanide & $10(7.2)$ \\
Hydrogen sulfide & $7(5.1)$ \\
Smoke & $7(5.1)$ \\
Unspecified gas $_{\text {Sulfur dioxide }}$ & $6(4.3)$ \\
Miscellaneous $^{\mathrm{a}}$ & $5(3.6)$ \\
Class total & $22(15.9)$ \\
\end{tabular}

${ }^{a}$ Includes dust, asbestos, carbon disulfide, chlorine, nitrogen oxides, petroleum vapors, arsine, carbon dioxide, chloramine, phosgene, phosphine, polyurethane vapors, radon, and silica 
Table 25 Household products

\begin{tabular}{ll}
\hline & $N(\%)$ \\
\hline Sodium hypochlorite $\leq 6 \%$ & $35(28.0)$ \\
Detergent pods & $26(20.8)$ \\
Cleaning solutions and disinfectants & $24(19.2)$ \\
Soaps and detergents unspecified & $13(10.4)$ \\
Household product unspecified $_{\text {Miscellaneous }^{\mathrm{a}}}$ & $11(8.8)$ \\
Class total & $16(12.8)$ \\
\end{tabular}

${ }^{a}$ Includes hair products, paints, ammonia $<10 \%$, hand sanitizer unspecified, deodorants/antiperspirants, dishwasher detergent, and sunscreens

of the class entries ( $51.7 \%$ ), followed by chromium, mercury, arsenic, and copper (Table 23). Carbon monoxide was the most common entry in the gases, irritants, vapors, and dust class (Table 24). A large fraction of the entries for the hydrocarbon class were unspecified (38.1\%); toluene and gasoline were the only specific agents with five or more reported cases. Brodifacoum, a 4-hydroxycoumarin vitamin $\mathrm{K}$ antagonist (anticoagulant) poison, was the most common rodenticide entry (23 cases). Only a limited number of herbicides and fungicides were reported (total $n=6$ ), with no agent with more than 2 entries. Organophosphates accounted for the majority of insecticide cases as indicated by entries for malathion, acephate, chlorpyrifos, and organophosphates unspecified, with several entries related to pyrethroids (permethrin, cypermethrin, pyrethrin unspecified). The caustic agent class also captured a broad range of agents, with only three individual agents with more than 5 occurrences (sodium hydroxide, hydrofluoric and hydrochloric acid), that combine to represent $36.3 \%$ of the class entries (see Supplementary Material).

Additional non-pharmaceutical agents are included across four classes: household products, caustics, other nonpharmaceuticals, and ingested foreign objects (Table 25 and Supplementary Material). Other than sodium hypochlorite $<6 \%$ in concentration ( $28 \%$ of class), the majority of agent entries are chemically nonspecific as compared to the pharmaceuticals; however, these agents were still associated with $1.0 \%$ of all agent entries (Table 8 ). In the class other nonpharmaceuticals, only "unspecified" agents had more than 3 entries, while batteries were the most commonly reported ingested foreign object.

\section{Clinical Signs and Symptoms}

At least one clinical sign or symptom was reported in 7512 $(81.9 \%)$ cases. These findings are summarized in Tables 26, $27,28,29$, and 30 , organized by either syndrome or organ system. Sedative-hypnotic and anticholinergic were the two most common toxidromes reported (both reported in over $5 \%$
Table 26 Toxidromes

\begin{tabular}{ll}
\hline & $N(\%)^{\mathrm{a}}$ \\
\hline Sedative-hypnotic & $631(6.9)$ \\
Anticholinergic & $467(5.1)$ \\
Opioid & $330(3.6)$ \\
Sympathomimetic & $232(2.5)$ \\
Serotonin syndrome & $210(2.3)$ \\
Sympatholytic & $36(0.4)$ \\
Alcoholic ketoacidosis & $26(0.3)$ \\
NMS & $15(0.2)$ \\
Washout syndrome & $11(0.1)$ \\
Anticonvulsant hypersensitivity & $8(0.1)$ \\
Overlap syndromes & $7(0.1)$ \\
Cholinergic & $5(0.05)$ \\
Fume fever & $<5(<0.05)$ \\
Total & $1980(21.6)$ \\
\hline
\end{tabular}

NMS neuroleptic malignant syndrome

${ }^{a}$ Percentage equals the number of cases reporting specific treatment relative to the total number of Registry cases in $2014(N=9172)$

of all cases), followed by opioid, sympathomimetic, and serotonin syndrome (Table 26). Tachycardia was the most common major vital sign abnormality ( $9.9 \%$ cases) followed by hypotension (5.7\% cases) and bradycardia (3.5\% cases) (Table 27). Neurological effects were encountered most frequently among all signs and symptoms. Coma or CNS depression was observed in 2641 (28.8 \%) cases (Table 28). Delirium, agitation, and rigidity/dystonia were relatively common as well, appearing in 10.7, 10.2, and $7.3 \%$ of cases, respectively. Among pulmonary signs or symptoms, respiratory depression occurred most frequently $(6.5 \%)$, while prolonged QTc ( $>500 \mathrm{~ms})$ or QRS (>120 ms) was the frequently reported cardiovascular effect (Table 29). All other individual signs or symptoms were reported in less than $6 \%$ of Registry cases (Table 30).

Table 27 Major vital sign abnormalities

\begin{tabular}{ll}
\hline & $N(\%)^{\mathrm{a}}$ \\
\hline Tachycardia $(\mathrm{HR}>140)$ & $910(9.9)$ \\
Hypotension (systolic $\mathrm{BP}<80 \mathrm{mmHg})$ & $525(5.7)$ \\
Bradycardia $(\mathrm{HR}<50)$ & $319(3.5)$ \\
$\begin{array}{l}\text { Hypertension }(\text { systolic } \mathrm{BP}>200 \mathrm{mmHg} \\
\quad \text { or diastolic } \mathrm{BP}>120 \mathrm{mmHg})\end{array}$ & $182(2.0)$ \\
Bradypnea $(\mathrm{RR}<10)$ & $149(1.6)$ \\
Hyperthermia (temp $\left.>105{ }^{\circ} \mathrm{F}\right)$ & $37(0.4)$ \\
Total & $1733(18.9)^{\mathrm{a}, \mathrm{b}}$ \\
\hline
\end{tabular}

$H R$ heart rate, $B P$ blood pressure

${ }^{a}$ Percentage equals the number of cases relative to the total number of Registry cases in $2014(N=9172)$

${ }^{\mathrm{b}}$ Total reflects cases reporting at least one major vital sign abnormality. Cases may be associated with more than one major vital sign abnormality 
Table 28 Clinical signs and symptoms - neurological

\begin{tabular}{ll}
\hline & $N(\%)^{\mathrm{a}}$ \\
\hline Coma/CNS depression & $2641(28.8)$ \\
Delirium & $979(10.7)$ \\
Agitation & $937(10.2)$ \\
Hyperreflexia/myoclonus/tremor & $667(7.3)$ \\
Seizures & $405(4.4)$ \\
Hallucinations & $276(3.0)$ \\
Dystonia/rigidity/extrapyramidal symptoms & $164(1.8)$ \\
Weakness/paralysis & $97(1.1)$ \\
Numbness/paresthesia & $74(0.8)$ \\
Peripheral neuropathy & $31(0.3)$ \\
Total & $4500(49.1)^{\mathrm{a}, \mathrm{b}}$ \\
\hline
\end{tabular}

CNS central nervous system

${ }^{a}$ Percentage equals the number of cases relative to the total number of Registry cases in $2014(N=9172)$

${ }^{\mathrm{b}}$ Total reflects cases reporting at least one neurological symptom. Cases may be associated with more than one neurological symptom

\section{Fatalities}

There were 89 fatalities reported in the Registry in 2014, comprising $0.97 \%$ of all cases (Tables 31 and 32, Supplementary Material). Forty-eight (53.4\%) of these cases were female. The average age of fatalities was 45.1 years, ranging from 8 weeks to 80 years of age. As in 2013, nonopioid analgesics and opioids were the most frequently reported agents among these cases. For cases reporting a single agent poisoning (38 cases or $42.7 \%$ of all fatalities), acetaminophen was the agent reported for all 12 analgesic-related events. One half of the six opioidrelated deaths were related to heroin, with the remainder of

Table 29 Clinical signs - cardiovascular and pulmonary

\begin{tabular}{ll}
\hline & $N(\%)^{\mathrm{a}}$ \\
\hline Cardiovascular & \\
Prolonged QTc $(\geq 500 \mathrm{~ms})$ & $265(2.9)$ \\
Prolonged QRS $(\geq 120 \mathrm{~ms})$ & $127(1.4)$ \\
Ventricular dysrhythmia & $69(0.8)$ \\
AV block (>1st degree) & $39(0.4)$ \\
Total & $417(4.5)^{\mathrm{b}}$ \\
Pulmonary & \\
Respiratory depression & $598(6.5)$ \\
Aspiration pneumonitis & $136(1.5)$ \\
Acute lung injury/ARDS & $75(0.8)$ \\
Asthma/reactive airway disease & $56(0.6)$ \\
Total & $772(8.4)^{\mathrm{b}}$ \\
\hline
\end{tabular}

$A R D S$ acute respiratory distress syndrome

${ }^{\text {a }}$ Percentage equals the number of cases reporting specific treatment relative to the total number of Registry cases in $2014(N=9172)$

${ }^{\mathrm{b}}$ Total reflects cases reporting at least one cardiovascular or pulmonary symptom. Cases may be associated with more than one symptom.
Table 30 Clinical signs - other organ systems

\begin{tabular}{ll}
\hline & $N(\%)^{\mathrm{a}}$ \\
\hline Metabolic & \\
Metabolic acidosis $(\mathrm{pH}<7.2)$ & $323(3.5)$ \\
Elevated anion gap $(>20)$ & $276(3.0)$ \\
Hypoglycemia (glucose $<50 \mathrm{mg} / \mathrm{dL})$ & $182(2.0)$ \\
Elevated osmole gap $(>20)$ & $40(0.4)$ \\
Total & $624(6.8)^{\mathrm{b}}$ \\
Gastrointestinal/hepatic & \\
Hepatotoxicity $(\mathrm{AST} \geq 1000 \mathrm{IU} / \mathrm{L})$ & $316(3.4)$ \\
Gastrointestinal bleeding & $48(0.5)$ \\
Corrosive injury & $35(0.4)$ \\
Pancreatitis & $31(0.3)$ \\
Intestinal ischemia & $8(0.1)$ \\
Total & $409(4.5)^{\mathrm{b}}$ \\
Hematological & \\
Coagulopathy $(\mathrm{PT}>15 \mathrm{~s})$ & $179(2.0)$ \\
Thrombocytopenia $(\mathrm{platelets}<100 \mathrm{~K} / \mu \mathrm{L})$ & $75(0.8)$ \\
Leukocytosis $(\mathrm{WBC}>20 \mathrm{~K} / \mu \mathrm{L})$ & $65(0.7)$ \\
Hemolysis $(\mathrm{Hgb}<10 \mathrm{~g} / \mathrm{dL})$ & $23(0.3)$ \\
Methemoglobinemia $(\mathrm{MetHgb} \geq 2 \%)$ & $13(0.1)$ \\
Pancytopenia & $13(0.1)$ \\
Coagulopathy $(\mathrm{PT}>15 \mathrm{~s})$ & $179(2.0)$ \\
Thrombocytopenia $(\mathrm{platelets}<100 \mathrm{~K} / \mu \mathrm{L})$ & $75(0.8)$ \\
Total & $306(3.3)^{\mathrm{b}}$ \\
Renal/musculoskeletal & $14(0.2)$ \\
Acute kidney injury $(\mathrm{creatinine}>2.0 \mathrm{mg} / \mathrm{dL})$ & $346(3.8)$ \\
Rhabdomyolysis $(\mathrm{CPK}>1000 \mathrm{IU} / \mathrm{L})$ & $317(3.5)$ \\
Total & $573(6.2)^{\mathrm{b}}$ \\
Dermatological & $122(1.3)$ \\
Rash & $76(0.8)$ \\
Blister/bullae & \\
Necrosis & \\
Angioedema & \\
\hline & \\
Total & \\
\hline
\end{tabular}

$P T$ prothrombin time, $W B C$ white blood cells, $H g b$ hemoglobin, $C P K$ creatinine phosphokinase

${ }^{\text {a }}$ Percentage equals the number of cases reporting specific treatment relative to the total number of Registry cases in 2014 ( $N=9172)$

${ }^{\mathrm{b}}$ Total reflects cases reporting at least one symptom in the category. Cases may be associated with more than one symptom

single agent opioid-related events due to methadone, oxycodone, and tramadol.

Among the 31 multiple agent fatalities (34.8\% of the total), 10 cases involved one or more opioids: oxycodone (4 cases), methadone (3 cases), and heroin ( 3 cases). Non-opioid analgesics were reported in seven multiple agent poisonings (primarily acetaminophen, nine cases).

In a substantial portion of cases, 22 of 89 (24.7\%), no agent was entered into the ToxIC Registry (see Supplementary Material). For the majority of this subset, 19 of 22 cases 

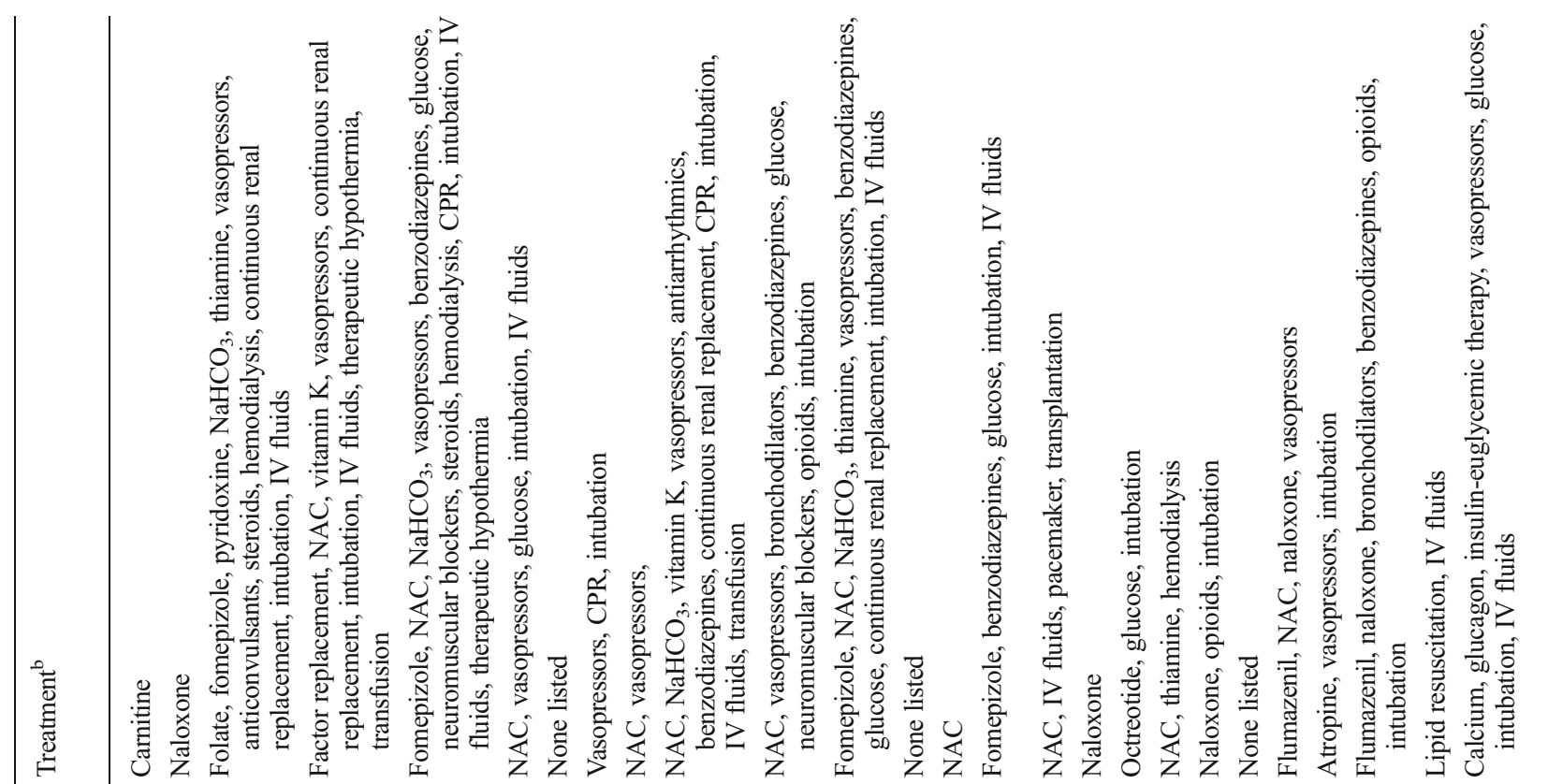

氞

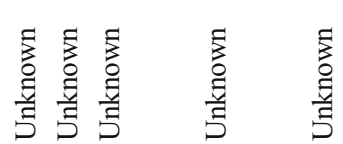

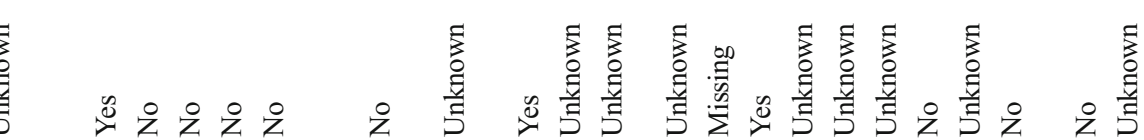
西

(25)

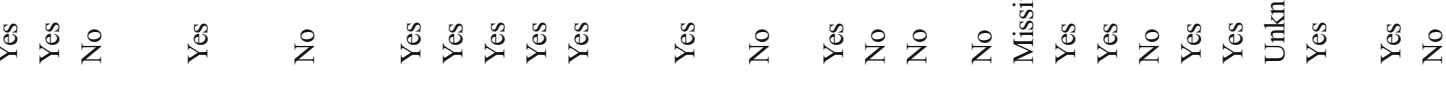

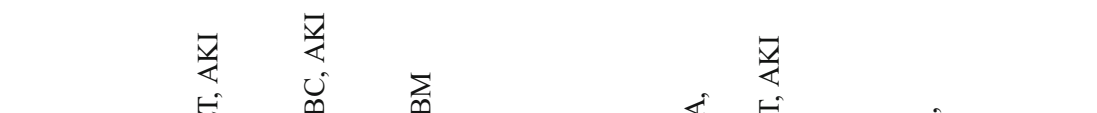

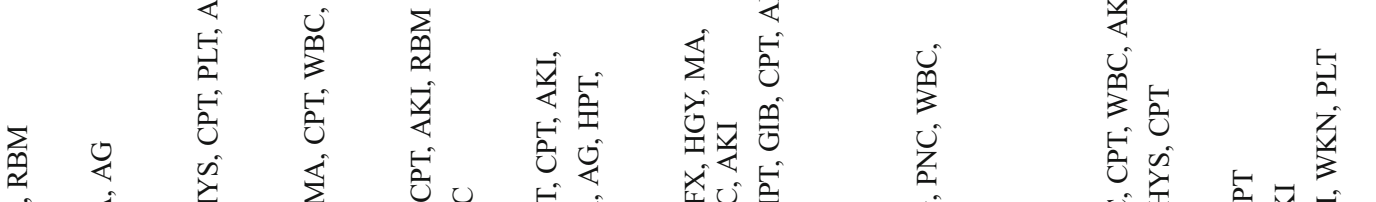

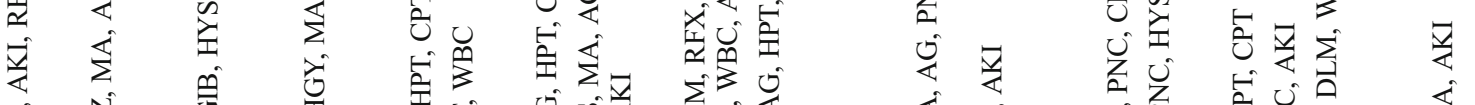

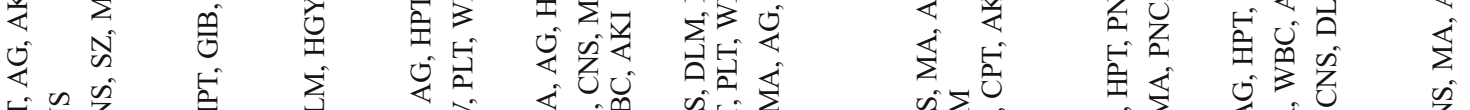

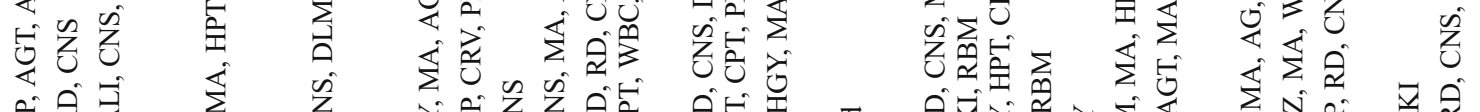
ले बे Uि

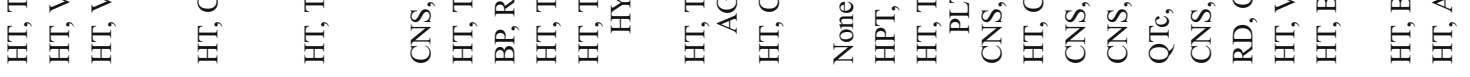




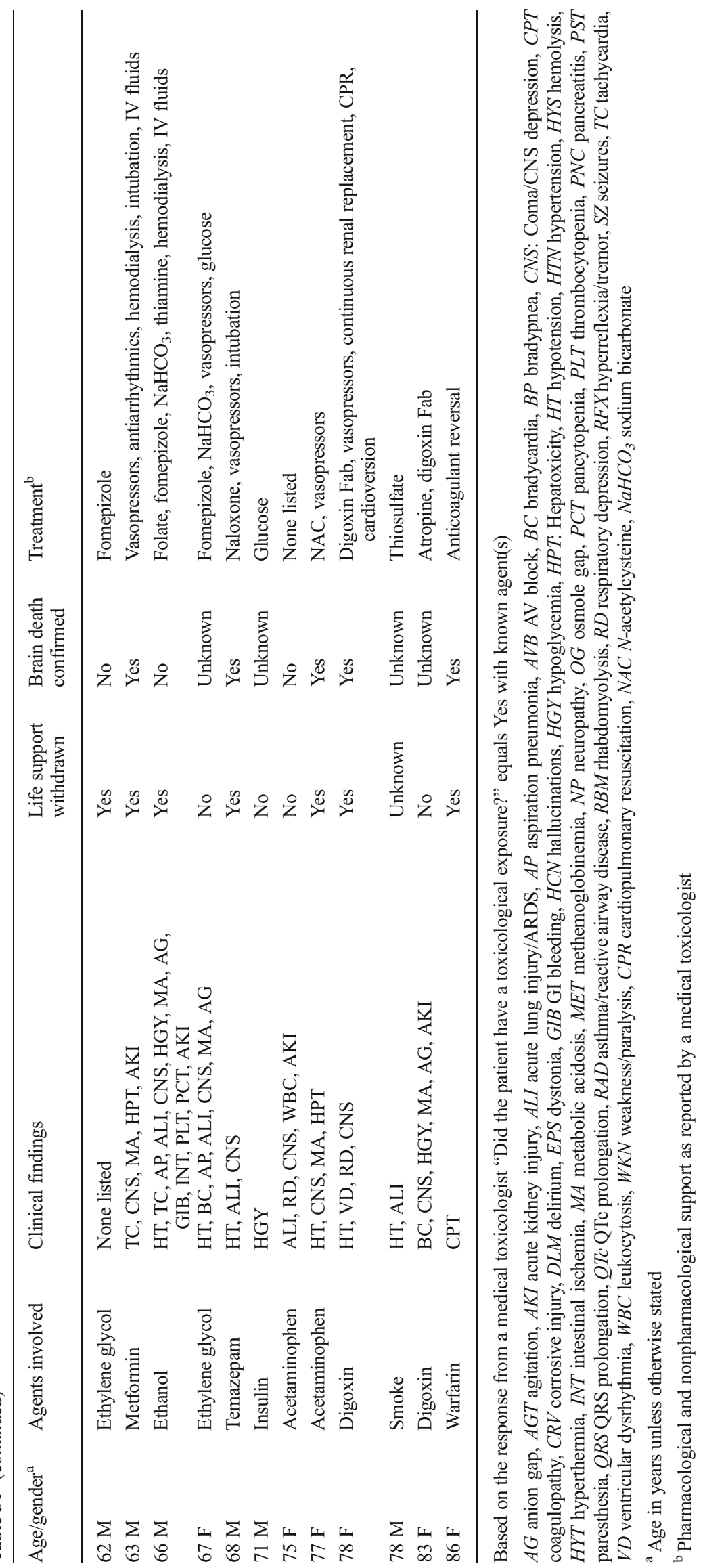




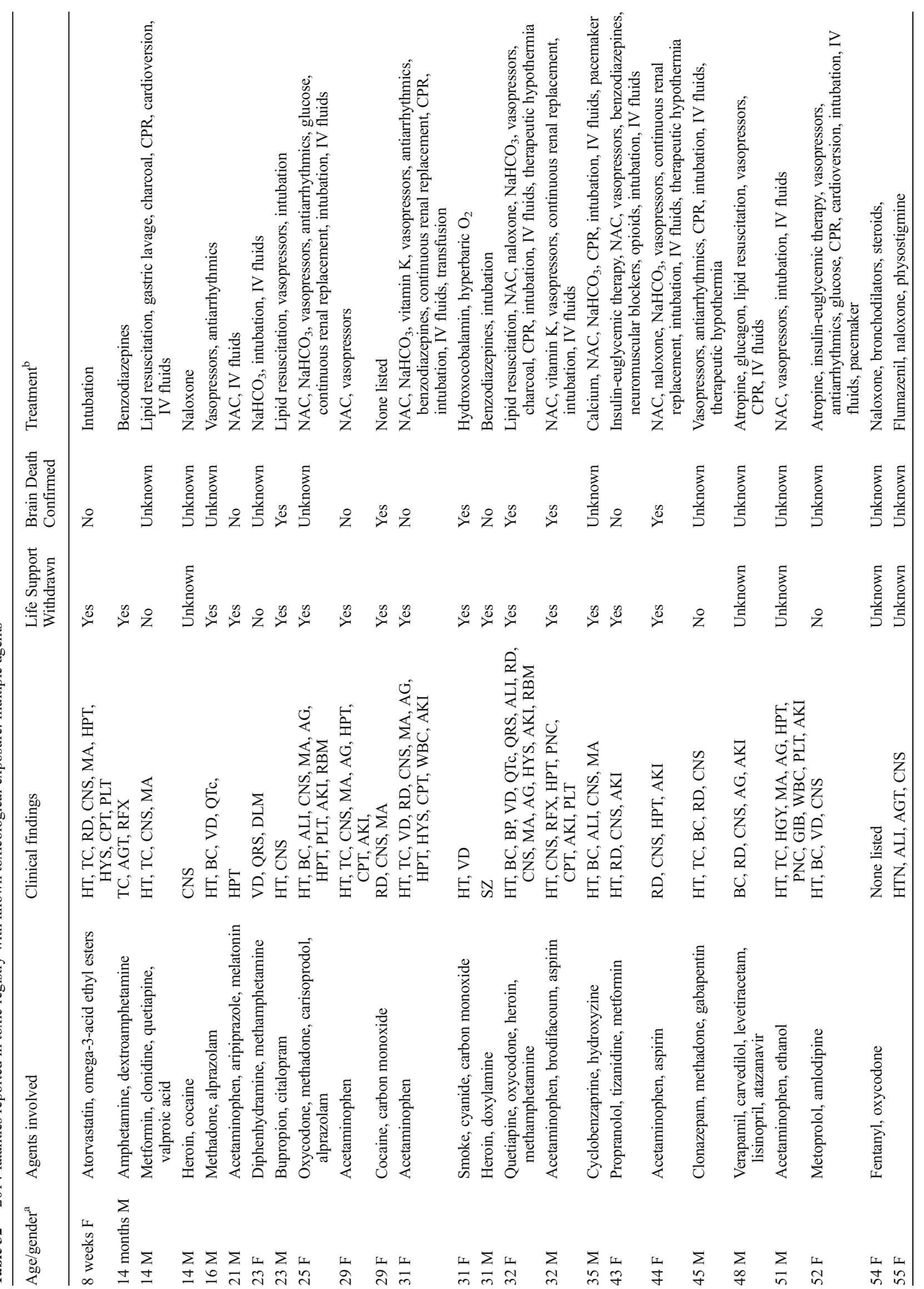




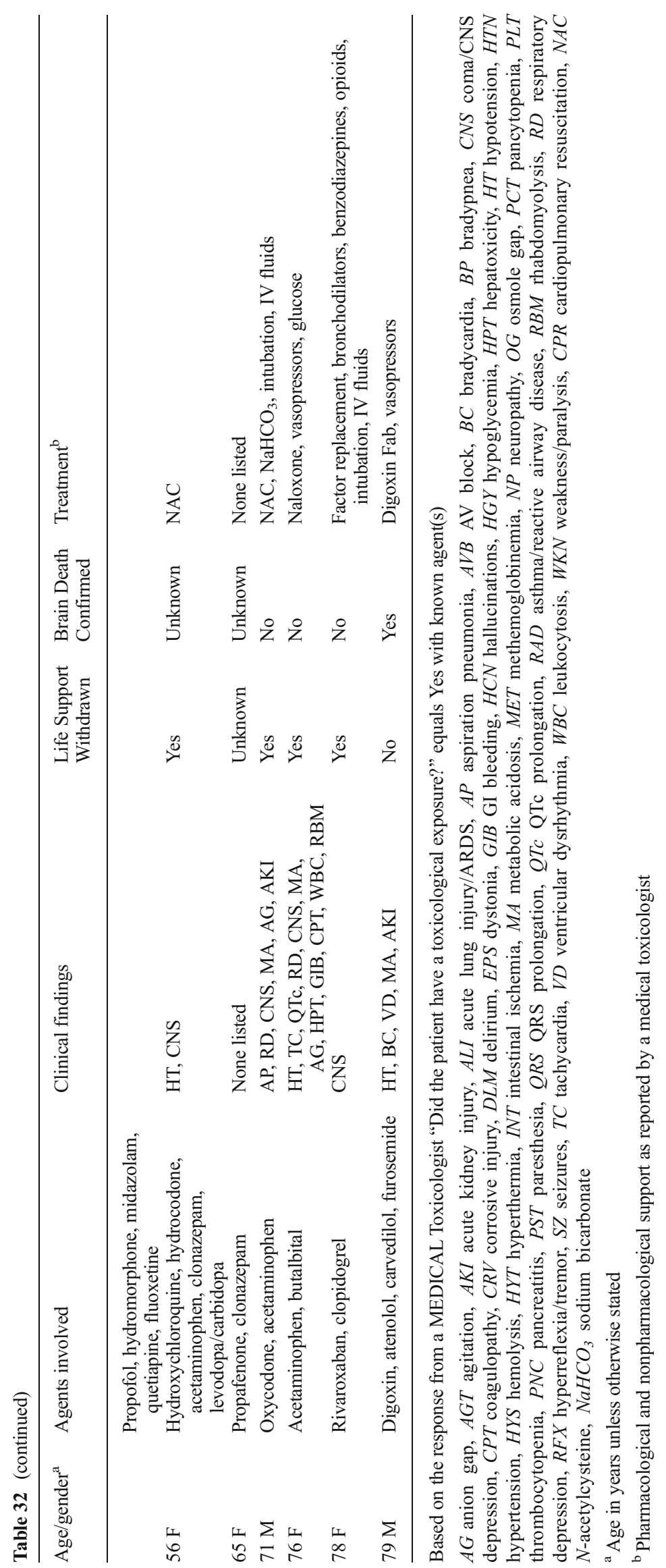


( $86.4 \%$ with no agent), the consulting/attending medical toxicologist determined that the patient did not have a toxicological exposure ( 7 cases), or it was unclear/unknown (12 cases); therefore, no agent information was entered. In the remaining three cases, the data was either missing or coded as "unknown agent" by the clinician.

In 57 cases (64\%), life support was withdrawn; among this latter subset, actual brain death was confirmed in 24 cases $(42.1 \%)$.

\section{Adverse Drug Reactions}

In 2014, 410 Registry cases (4.5\%) reported the involvement of an adverse drug reaction (ADR). During 2014, Registry data fields were expanded to include a separate field to identify ADRs from among the reasons for a toxicological consultation. A total of 194 drugs or substances were mentioned at least once with the aid of this additional indicator field. Table 33 lists the 16 most frequently encountered drugs associated with ADRs (single and multiple drug exposure). The overall findings are similar to the ADR summary from the 2013 Registry report [5]. Lithium remains the most frequently cited drug associated with ADRs. Likewise, the most frequently encountered agent classes (non-opioid analgesics, sedative-hypnotics, opioids) are relatively underrepresented in Table 33, with psychiatric medications (antipsychotics $14.6 \%$, antidepressants $19.3 \%$ ) and cardiovascular

Table 33 Most common drugs associated with ADRs

\begin{tabular}{ll}
\hline & $N(\%)^{\mathrm{a}}$ \\
\hline Lithium & $45(11.0)$ \\
Digoxin & $29(7.1)$ \\
Valproic acid & $16(3.9)$ \\
Phenytoin & $14(3.4)$ \\
Quetiapine & $12(2.9)$ \\
Citalopram & $11(2.7)$ \\
Tramadol & $11(2.7)$ \\
Bupropion & $10(2.4)$ \\
Risperidone & $10(2.4)$ \\
Trazodone & $10(2.4)$ \\
Aripiprazole & $8(2.0)$ \\
Fentanyl & $8(2.0)$ \\
Glipizide & $8(2.0)$ \\
Methadone & $8(2.0)$ \\
Metoprolol & $8(2.0)$ \\
Sertraline & $8(2.0)$ \\
Total & $216(36.4)$
\end{tabular}

${ }^{\text {a }}$ Percentages are out of the total number of all drugs reported involved in adverse drug reactions (ADRs); 410 ADRs, with 593 individual agents; $4.5 \%$ of registry cases in 2014 reported as a ADR medications (17.6\%) cited more frequently. The relative pattern changes somewhat if only single drug exposure events are considered (259 or $63.2 \%$ of all ADRs). Lithium (13.5\%), digoxin $(9.3 \%)$, and phenytoin and valproic acid (both $<5 \%$ ) are the four most common individual medications, with cardiovascular $(15.8 \%)$, lithium $(13.5 \%)$, anticonvulsants $(10.8 \%)$, and antipsychotics $(8.9 \%)$ as the most common classes.

\section{Treatment}

Specific treatment was rendered in $62.3 \%$, with 5715 Registry cases reporting more than one treatment modality. There were a total of 2962 instances of antidote administration, accounting for $51.8 \%$ of all treatments reported; $4.2 \%$ of all Registry cases received more than one antidotal therapy for a given event. $\mathrm{N}$-acetylcysteine and naloxone/nalmefene collectively comprised over half of all antidotal treatment (Table 34). With the exception of sodium bicarbonate, all other antidotes were used relatively infrequently, with each individual drug accounting for less than $10 \%$ of antidote administrations. Antivenom usage was uncommon, being given in only 197 $(2.1 \%)$ of all Registry cases, with polyvalent anti-Crotalidae Fab accounting for a large majority of antivenom treatments (93.4\%) (Table 35).

In the case of pharmacological support, $5.2 \%$ of the Registry cases indicated that more than one form was used for the given toxic event (Table 36). Benzodiazepines and opioids were utilized most frequently, combining for approximately two thirds of all treatments rendered. There were 2867 nonpharmacological therapies given, with $5.8 \%$ of cases receiving more than one treatment modality (Table 37). Intravenous fluid resuscitation and mechanical ventilation management accounted for a large majority of treatments, 67.6 and $27.8 \%$, respectively. The remaining non-pharmacological supportive care each accounted for less than $2 \%$ of treatments rendered. Chelation was reported for only 21 Registry cases, with 2 receiving multiple chelation modalities. Dimercaptosuccinic acid (DMSA) and ethylenediaminetetraacetic acid (EDTA) accounted for 18 of the 23 chelation therapies reported (Table 38).

There were 409 recorded decontamination therapies across 372 cases, indicating that 37 cases received multiple treatments (Table 39). Activated charcoal was the most frequently reported decontamination modality applied (79.5\%). There were 245 separate uses of enhanced elimination techniques, with only $0.3 \%$ of all registry cases receiving multiple forms of enhanced elimination (Table 40). Renal replacement therapy, either hemodialysis or continuous renal replacement (e.g., CVVH), accounted for $70.6 \%$ of enhanced elimination therapy. 
Table 34 Antidotal therapy

\begin{tabular}{ll}
\hline & $N(\%)^{\mathrm{a}}$ \\
\hline$N$-acetylcysteine & $921(31.1)$ \\
Naloxone/nalmefene & $605(20.4)$ \\
Sodium bicarbonate & $322(10.9)$ \\
Physostigmine & $156(5.3)$ \\
Thiamine & $119(4.0)$ \\
Fomepizole & $90(3.0)$ \\
Flumazenil & $81(2.7)$ \\
Glucagon & $80(2.7)$ \\
Calcium & $77(2.6)$ \\
Folate & $74(2.5)$ \\
Octreotide & $67(2.3)$ \\
Atropine & $51(1.7)$ \\
Cyproheptadine & $49(1.7)$ \\
Vitamin K & $45(1.5)$ \\
Insulin-euglycemic therapy & $41(1.4)$ \\
L-Carnitine & $38(1.3)$ \\
Fab for digoxin & $35(1.2)$ \\
Lipid resuscitation & $33(1.1)$ \\
Pyridoxine & $17(0.6)$ \\
Bromocriptine & $12(0.4)$ \\
Dantrolene & $11(0.4)$ \\
Hydroxocobalamin & $11(0.4)$ \\
2-PAM & $7(0.2)$ \\
Anticoagulant reversal therapy & $4(0.1)$ \\
Thiosulfate & $4(0.1)$ \\
Ethanol & $3(0.1)$ \\
Coagulation factor replacement & $3(0.1)$ \\
Methylene blue & $3(0.1)$ \\
Nitrites & $3(0.1)$ \\
Total & $2962(100)$ \\
\hline & \\
\hline
\end{tabular}

${ }^{\text {a }}$ Percentages are out of the total number of antidotes administered (2962); $4.2 \%$ of registry cases received more than one antidote

\section{Discussion}

This report of the ACMT ToxIC Registry serves as an overview of cases involving medical toxicology consultations reported in 2014. In its fifth year, the Registry continues to grow in both the number of reported cases and participating

Table 35 Antivenom therapy

\begin{tabular}{ll}
\hline & $N(\%)^{\mathrm{a}}$ \\
\hline Polyvalent anti-Crotalidae Fab fragments & $184(93.4)$ \\
Spider antivenom & $6(3.0)$ \\
Other snake antivenom & $4(2.0)$ \\
Scorpion antivenom & $3(1.5)$ \\
Total & $197(100)$ \\
\hline
\end{tabular}

${ }^{a}$ Percentages are out of the total number of antivenom treatments administered (197)
Table 36 Supportive care-pharmacological

\begin{tabular}{ll}
\hline & $N(\%)^{\mathrm{a}}$ \\
\hline Benzodiazepines & $1624(7.1)$ \\
Opioids & $261(9.2)$ \\
Vasopressors & $239(8.4)$ \\
Antipsychotics & $186(6.5)$ \\
Glucose (concentration>5\%) & $165(5.8)$ \\
Anticonvulsants & $78(2.7)$ \\
Neuromuscular blockers & $66(2.3)$ \\
Albuterol (or other bronchodilator) & $63(2.2)$ \\
Corticosteroids & $49(1.7)$ \\
Antiarrhythmics & $42(1.5)$ \\
Antihypertensives & $35(1.2)$ \\
Beta blockers & $27(0.9)$ \\
Vasodilators & $8(0.3)$ \\
Total & $2843(100)$
\end{tabular}

${ }^{a}$ Percentages are out of the total number of treatments administered (2843); $5.2 \%$ of registry cases received more than one form of pharmacological treatment

institutions (Fig. 1, Tables 1 and 2). In 2014, many of the observed percentages of type of consultation, reason for consultation, as well as general agent class reported remain similar to those found in prior years [2-5]. Intentional pharmaceutical exposure remains the most frequent cause of consultations, with analgesics, sedative-hypnotic agents, opioids, and antidepressants continuing to constitute the most frequently encountered agent classes.

However, the rank order based on relative proportion of these classes has varied somewhat over the 5-year period.

Table 37 Supportive care-non-pharmacological

\begin{tabular}{ll}
\hline & $N(\%)^{\mathrm{a}}$ \\
\hline IV fluid resuscitation & $1937(67.6)$ \\
Intubation/ventilatory management & $796(27.8)$ \\
CPR & $40(1.4)$ \\
Hyperbaric oxygen & $21(0.7)$ \\
Transfusion & $21(0.7)$ \\
Pacemaker & $15(0.5)$ \\
Therapeutic hypothermia & $13(0.5)$ \\
Cardioversion & $11(0.4)$ \\
ECMO & $7(0.2)$ \\
Organ transplantation & $4(0.1)$ \\
Aortic balloon pump & $1(0.0)$ \\
Bypass & $1(0.0)$ \\
Total & $2867(100)$ \\
\hline$C P R$ cardiopulmonary resuscitation, ECMO extracorporeal membrane \\
oxygenation \\
a Percentages are out of the total number of treatments administered \\
(2867); 5.8 \% of registry cases received more than one form of \\
nonpharmacological treatment
\end{tabular}


Table 38 Chelation therapy

\begin{tabular}{ll}
\hline & $N(\%)^{\mathrm{a}}$ \\
\hline DMSA & $11(47.8)$ \\
EDTA & $7(30.4)$ \\
Dimercaprol (BAL) & $4(17.4)$ \\
Deferoxamine & $1(4.3)$ \\
Total & $23(100)$ \\
\hline
\end{tabular}

DMSA dimercaptosuccinic acid, EDTA ethylenediamine-tetraacetic acid

${ }^{\text {a }}$ Percentages are out of the total number of chelation treatments administered (23)
As the number of data years collected increase, the ability to observe real changes or trends will continue to improve. The degree to which this variability reflects an actual change in the affected populations' exposure (or type of use) versus operational or other characteristic changes in the participating institutions cannot be determined from a single broad, descriptive review based on single variables. The Registry data can be used to identify more refined areas for hypothesis generation, and provide starting points for initiating additional observation or clinical research by specific type of encounter, agent, or treatment.

As a nonpopulation-based surveillance system, the ToxIC Registry is unable to produce a weighted rate based on either total population covered, or total poisoning/clinical cases for a given medical catchment or geographic area. The Registry nonetheless provides a standardized and detailed view of the relative occurrence in the type of poisonings and other clinical encounters due to both acute and chronic toxic exposures severe enough to require clinical intervention from a medical toxicologist. This feature provides an opportunity to identify changes in exposure, as well as clinical care and practice over time, for the most severe, subset of poisoning cases. However, in order to utilize such data obtained through any disease and clinical surveillance system requires vigilance in the specific data collected, primarily through design of the data collection tool and quality improvement activities.

Table 39 Decontamination

\begin{tabular}{ll}
\hline & $N(\%)^{\mathrm{a}}$ \\
\hline Activated charcoal & $325(79.5)$ \\
Whole bowel irrigation & $46(11.2)$ \\
Gastric lavage & $23(5.6)$ \\
External irrigation & $15(3.7)$ \\
Total & $409(100)$ \\
\hline
\end{tabular}

${ }^{\text {a }}$ Percentages are out of the total number of treatments administered (409); 37 registry cases received more than one form of decontamination
Table 40 Enhanced elimination

\begin{tabular}{ll}
\hline & $N(\%)^{\mathrm{a}}$ \\
\hline Hemodialysis (toxin removal) & $74(30.2)$ \\
Urinary alkalinization & $56(22.9)$ \\
Hemodialysis (other indication) & $50(20.4)$ \\
Continuous renal replacement therapy & $49(20.0)$ \\
Multiple-dose activation charcoal & $14(5.7)$ \\
Exchange transfusion & $2(0.8)$ \\
Total & $245(100)$ \\
\hline
\end{tabular}

${ }^{\text {a }}$ Percentages are out of the total number of treatments administered (245); 27 registry cases received more than one form of enhanced elimination

Reporting bias is a potential limitation of any database dependent on voluntary reporting. However, all participating sites agree, as a condition of participation, that all of their consultations will be entered into the Registry, thus minimizing such bias. In the initial years of the Registry, data quality had been the responsibility of the participating sites, which could lead to missing, inconsistent, or unclear data capable of leading to underreporting. In 2014, the ToxIC Registry initiated a centralized quality assurance review of Registry data, to help reduce issues such as incorrectly coded or absent data fields. The degree of detail concerning therapy was also variable. While it is likely that many fatality cases received certain aspects of critical care, such as intubation or IV fluids, documentation of therapy was relatively minimal in some cases. With the initiation of a more centralized quality improvement program, the ToxIC Registry will work toward reducing issues such as incorrectly coded or absent data fields, and programming changes to help differentiate missing/ skipped data fields from "not applicable/none." This has now been applied in several areas including clinical signs and symptoms, treatment, and outcome (death) with the aim to improve case completeness and data quality in future years.

In addition, while it may be inferred from the type of treatment rendered (Tables 34, 35, 36, 37, 38, 39, and 40), the Registry lacks a mechanism to directly describe the relative severity of toxicity (aside from fatality) in any specific case. Currently, a study is underway, and data is being collected, aimed at designing a severity scoring system for ToxIC. Furthermore, by not being populationbased, the ToxIC Registry has a specific ascertainment bias, which is present by design. The key inclusion criterion for entry into the Registry is the consultation by a medical toxicologist. Thus, Registry cases represent patients for whom there was a concern for significant toxicity. Cases of no, or mild, toxicity are likely to be underrepresented.

In response to data from earlier years, several changes were initiated in 2014 including improved demographic information on race and ethnicity to better identify subgroups 
potentially at higher risk or specific toxic encounters. Unfortunately, this variable was initiated mid-year with limited detailed response, with a large proportion of participating institutions indicating "Unknown" for the two variables: $32.8 \%$ race and $29.5 \%$ ethnicity (Table 4 ). In order to provide consistent and useful information, the Registry will need to continue to actively inform and follow up with sites to improve the data quality on this important social indicator.

Another 2014 data change focused on refining the information collected on events related to intentional pharmaceutical use (52.4\% cases in 2014), specifically by adding an additional information requirement to detail the presence of attempt at self-harm in any intentional exposure. This information was provided for the majority of relevant cases (Table 7), therefore enabling future study into agent type, treatment provided, and patient outcome in cases of self-harm with suicidal intent.

Also in 2014, concerns around another type of pharmaceutical exposure, specifically adverse drug reactions (ADRs), continue. Over the year, 410 events were identified as ADRs, and another 175 as adverse drug events (ADEs), 4.5 and $1.9 \%$, respectively (data not presented). However, concerns persist to the relative completeness of the data field. In the future, the Registry will include more stringent data entry requirements, including that each case must be documented as an ADR (undesirable effect medication at a normal dose) or a medication error, in order to submit a case to the Registry. Additional question subfields related to type of event (e.g., exaggeration, continuing action, etc.), or error (e.g., administering, dosing, dispensing error, etc.), type of intervention, and strength of causality are now required. By better elucidating ADR-related events, the Registry aims to recreate a large, detailed case set for more effective descriptive analysis and to determine the relative engagement, and influence of, medical toxicologists in these cases.

Fatality data has also been expanded to include the withdrawal of care in poisoning cases. Information regarding withdrawal of care collected in this and future Registry reports will help increase understanding of a controversial issue for a subset of severe poisoning and other exposure events [8].

\section{Conclusions}

The majority of cases requiring medical toxicology consultation in 2014 involved intentional or unintentional exposure to pharmaceutical products. Non-opioid analgesics, sedative-hypnotic agents, and opioids remain the most commonly encountered agent classes. Though nearly two thirds of patients required some form of medical treatment, fatalities were uncommon.

Acknowledgments The authors express their sincere gratitude to the staff at the American College of Medical Toxicology (ACMT) for supporting the ToxIC Registry project. We very much appreciate the contribution to the Registry from each of the ToxIC sites providing cases in

2014. The following is a list of the principal coordinators from each site:

Albuquerque, NM

Steven Seifert, MD

Atlanta, GA

Ziad Kazzi, MD

Boston, MA (Beth Israel Deaconess Medical Center)

Michael Ganetsky, MD

Boston, MA (Children's Hospital of Boston)

Michele Burns, MD

Charlotte, NC

Michael Beuhler, MD

Charlottesville, VA

Joshua D. King, MD

Cincinnati, $\mathrm{OH}$

Shan Yin, MD

Dallas, TX

Kurt Kleinschmidt, MD

Paul Wax, MD

Denver, CO

Jeffrey Brent, MD, PhD

Eric Lavonas, MD

Evanston, IL

Jerrold Leikin, MD

Fresno, CA

Rais Vohra, MD

Grand Rapids, MI

Bryan Judge, MD

Brad Riley, MD

Haifa, Israel

Didi Bentur, MD

Harrisburg, PA

Phil Moore, MD

Hartford, CT

Mark Neavyn, MD

Houston, TX

Spencer Greene, MD

Indianapolis, IN

Daniel Rusyniak, MD

Kansas City, MO

Jennifer Lowry, MD

Adam Algren, MD

Loma Linda, CA

Tammy Phan, MD

Los Angeles, CA

Michael Levine, MD

Manhasset, NY

Josh Nogar, MD

Melbourne, Australia

Anselm Wong, MD

Shaun Greene, MD

Milwaukee, WI

Mark Kostic, MD

David Gummin, MD

Morristown, NJ

Diane Calello, MD

Oliver Hung, MD

New Brunswick, NJ

Ann-Jeannette Geib, MD 
New York, NY (Mt. Sinai Hospital)

Stephanie Hernandez, MD

New York, NY (NYU Langone Medical Center)

Silas Smith, MD

Newark, NJ

Steven Marcus, MD

Omaha, NE

Ronald Kirschner, MD

Philadelphia (Einstein Medical Center)

Adam Rowden, MD

Philadelphia, PA (Hahnemann University Hospital)

David Vearrier, MD

Rita McKeever, MD

Phoenix, AZ

Michelle Ruha, MD

Pittsburgh, PA

Anthony Pizon, MD

Portland, OR

Robert Hendrickson, MD

Nate McKeown, MD

Richmond, VA

Brandon Wills, MD

Kurt Cumpston, MD

Riyadh, Saudi Arabia

Mohammed Alhelail, MD

Rochester, NY

Timothy Wiegand, MD

Salt Lake City, UT

E. Martin Caravati, MD

San Antonio, TX

Vik Bebarta, MD

Joseph Maddry, MD

San Francisco, CA

Derrick Lung MD

Craig Smollin MD

St. Louis, MO

Thomas Kibby, MD

Evan Schwarz MD

St. Paul, MN

Samuel Stellpflug, MD

Kristin Engebretsen, MD

Sydney, Australia

Naren Gunja, MD

Syracuse, NY
Ross Sullivan, MD

Toronto, Canada

Yaron Finkelstiein, MD

Worcester, MA

Sean Rhyee, MD

Conflict of Interest The authors declare that they have no competing interests.

Funding Sources This study received funding from the NIH National Institute of Neurological Disorders and Stroke 3U01NS083452-01 (Bird, SB), NIH National Institute on Drug Abuse 1R56DA38366 (Boyer, EW/ Carlson, RG) and 1R01DA037317-01 (Manini, A), and BTG International Inc.

\section{References}

1. Wax PM, Kleinschmidt KC, Brent J. The Toxicology Investigators Consortium (ToxIC) Registry. J Med Toxicol. 2011;7(4):259-65.

2. Brent J, Wax PM, Schwartz T, et al. The Toxicology Investigators Consortium Case Registry - the 2010 experience. J Med Toxicol. 2011;7(4):266-76.

3. Wiegand TJ, Wax PM, Schwartz T, et al. The Toxicology Investigators Consortium Case Registry - the 2011 experience. J Med Toxicol. 2012;8(4):360-77.

4. Wiegand T, Wax P, Smith E, et al. The Toxicology Investigators Consortium Case Registry - the 2012 experience. J Med Toxicol. 2013;9(4):380-404.

5. Rhyee SH, Farrugia L, Wiegand T, Smith EA, Wax PM, Brent J, et al. The Toxicology Investigators Consortium Case Registry-the 2013 experience. J Med Toxicol. 2014;10(4):342-59.

6. Blake A, Froberg BA, Levine M, Beuhler MC, Judge BS, Moore PW, et al. Acute methylenedioxypyrovalerone toxicity. J Med Toxicol. 2014. doi:10.1007/s13181-014-0446-8.

7. Watkins JW, Schwarz ES, Arroyo-Plasencia AM, Mullins ME, on behalf of the Toxicology Investigators Consortium. The use of physostigmine by toxicologists in anticholinergic toxicity. J Med Toxicol. 2014. doi:10.1007/s13181-014-0452-x.

8. Neavyn MJ. Suicide and the surrogate. J Med Toxicol. 2014;10(1): $3-6$ 